\title{
Cholinergic neuromodulation of inhibitory interneurons facilitates functional integration in whole-brain models
}

\author{
Carlos Coronel-Oliveros ${ }^{1,2}$, Rodrigo Cofré ${ }^{3}$, Patricio Orio ${ }^{1,4^{*}}$ \\ 1 Centro Interdisciplinario de Neurociencia de Valparaíso, Universidad de Valparaíso, \\ Valparaíso, Chile \\ 2 Programa de Doctorado en Ciencias, mención Biofísica y Biología Computacional, \\ Universidad de Valparaíso, Valparaíso, Chile \\ 3 CIMFAV-Ingemat, Facultad de Ingeniería, Universidad de Valparaíso, Valparaíso, \\ Chile \\ 4 Instituto de Neurociencias, Facultad de Ciencias, Universidad de Valparaíso, \\ Valparaíso, Chile \\ *patricio.orio@uv.cl
}

\begin{abstract}
Segregation and integration are two fundamental principles of brain structural and functional organization. Neuroimaging studies have shown that the brain transits between different functionally segregated and integrated states, and neuromodulatory systems have been proposed as key to facilitate these transitions. Although

computational models have reproduced the effect of neuromodulation at the whole-brain level, the role of local inhibitory circuits and their cholinergic modulation has not been studied. In this article, we consider a Jansen \& Rit whole-brain model in a network interconnected using a human connectome, and study the influence of the cholinergic and noradrenergic neuromodulatory systems on the segregation/integration balance. In our model, a newly introduced local inhibitory feedback enables the integration of whole-brain activity, and its modulation interacts with the other neuromodulatory influences to facilitate the transit between different functional states. Moreover, the new proposed model is able to reproduce an inverted-U relationship between noradrenergic modulation and network integration. Our work proposes a new possible mechanism behind segregation and integration in the brain.
\end{abstract}

\section{Introduction}

Integration and Segregation of brain activity are nowadays two well-established brain organization principles $[1-4]$. Functional segregation refers to the existence of specialized brain regions, allowing the local processing of information. Integration coordinates these local activities in order to produce a coherent response to complex tasks or environmental contexts [1,2]. Both segregation and integration are required for the coherent global functioning of the brain; the balance between them constitutes a key element for cognitive flexibility, as highlighted by the theory of coordination dynamics 5,6 .

From a structural point of view, the complex functional organization of the brain is possible thanks to an anatomical connectivity that combines both integrated and segregated network characteristics, having small-world and modular properties [7]. In 
spite of this structural connectivity (SC) remaining fixed over short time scales, different patterns of functional connectivity (FC) can be observed during the execution of particular behavioral tasks [2]. Moreover, functional Magnetic Resonance Imaging (fMRI) neuroimaging studies show that during a resting state the FC is not static, but rather evolves over the recording time. The non-stationarity of functional connectivity, referred as Functional Connectivity Dynamics (FCD), captures the variable nature of the brain dynamics [8, 9]. In this context, an interesting question emerges: How does the brain manage to produce dynamical transitions between different functional states from a rigid anatomical backbone?

Neuromodulatory systems provide a biophysical mechanism that enhances the dynamical flexibility. It is thought that functional integration arises by the increase of excitability and selectivity of neuronal populations, and a recent hypothesis proposed by [10 argues that neuromodulation allows the transition between integrated and segregated states, manipulating the neural gain function [11. Indeed, the cholinergic system increments the overall excitability [12,13], and consequently rises population activity above noise, a mechanism referred as response gain [11]. The increase in signal-to-noise ratio, especially in brain areas that are close to each other, promotes segregation when considering the response gain by itself $[10$. On the other hand, the noradrenergic system increases the reponsivity (or selectivity) of neuronal populations to input-driven activity respect to spontaneous activity [14 16 ] and filters out noise 17 , a mechanism called filter gain 11. This effect is more pronounced between distant brain regions, in which structural connectivity is relatively low, promoting functional integration 10. In reality, a complex interaction between the cholinergic and noradrenergic system seems to manage the balance between integration and segregation. Using a whole-brain model, [18 showed that the inverted-U relationship between neuromodulation and integration, which has been reported in the literature 19, can be reproduced manipulating the effects of cholinergic and noradrenergic systems on neural gain. In this model, integration is not promoted by any neuromodulatory system by itself, but emerges by the combined action of both systems: the cholinergic and noradrenergic systems do not operate in an antagonistic fashion.

There are still unanswered questions about the specific effects of neuromodulation on integration and segregation. The overall increase of excitability, mediated by the cholinergic system, affects both the excitatory and inhibitory neuronal populations, effect well known at the meso-scale level [11,20 23. Experimental research points out that the cholinergic system, through both nicotinic and muscarinic receptors, boosts the signal-to-noise ratio in two principal ways [11,22]: first, increasing the excitability of pyramidal neurons 23 25], and second, enhancing the activity and firing rates of dendritic-targeting GABAergic interneurons, an effect that promotes intra-columnar inhibition, reducing the local excitatory feedback to pyramidal neurons $23,26,27$. Consequently, pyramidal neurons become more responsive to stimulus from other distant regions respect to the stimulus of its own cortical column 21,22, 24. The particular effect of the cholinergic system in excitatory neurons was one of the focus of the whole-brain simulation work by $[18$. However, the cholinergic modulation of inhibitory interneurons and its effect on the segregation/integration balance has not been analyzed at the whole-brain level, and comprises the main focus of the present work.

Here, we use an in silico approach to analyze the effect of neuromodulatory systems on functional integration in the brain, focusing on the cholinergic action in inhibitory interneurons. Whole-brain computational models can reproduce statistical features of neuroimaging signals from human brains 28,29], providing a tool to explore the computational and biophysical mechanisms that underlie the organization principles of integration and segregation [18,30,31]. We combined a real human structural connectivity with the Jansen \& Rit neural mass model of cortical columns [32, widely 
used to reproduce electroencephalography (EEG) signals in the healthy and pathological brain 33 35]. fMRI-blood-oxygen-level dependent (BOLD) signals were generated from the firing rates of pyramidal neurons to quantify integration and segregation in the functional connectivity matrices derived from the BOLD-like signals using a graph theoretical approach.

The neuromodulation was discerned in three components. First, we included an "excitatory gain", which increases the inter-columnar coupling. This gain mechanism is mediated by the action of the cholinergic system in pyramidal neurons, principally but not exclusively, and increments pyramidal excitability 10, 11, 22. Second, we added an "inhibitory gain", also mediated by the cholinergic system, that controls the inputs from inhibitory to excitatory interneurons and reduces the local feedback excitation. This additional connection, well described in cortical columns 36, 37, represents a modification of the original neural mass model proposed by $[32]$. Finally, we incorporated a "filter gain", that increments the pyramidal neurons sigmoid function slope [1]. This last gain mechanism is mediated by the noradrenergic system; it acts as a filter, decreasing (increasing) the responsivity to weak (strong) stimuli [15, 17], boosting signal-to-noise ratio and promoting integration [10].

Our results show, in the context of a whole-brain model, that the control of local feedback excitation, mediated by the action of the cholinergic system on inhibitory interneurons, is necessary for the modulation of the segregation/integration balance by the other systems. This constitutes a step forward from the neuromodulatory framework proposed by [10, including the role of a second cholinergic target and also highlighting the role of a homeostatic inhibitory feedback. We also describe that integration is accompanied by an increment in the signal-to-noise ratio, and a reduction of dynamical variability captured by the FCD analysis. Our work sheds light in how meso-scale properties, such as local inhibition, shape statistical network features at the macro-scale level.

\section{Results}

We assessed the effect of the neuromodulatory systems using a whole-brain neural mass model of brain activity. In the model, each node corresponds to a brain area and is represented by a neural mass consisting of three populations [32]: pyramidal neurons, excitatory interneurons, and inhibitory interneurons (Fig 1A. Based on Silberberg \& Markram [36] and Fino et al. 37], we have added a connection from inhibitory interneurons from excitatory interneurons (dotted line in Fig 1A), allowing us to study the effect of its modulation by cholinergic influences (see below). The nodes are connected through a weighted and undirected structural connectivity matrix derived from human data 38, parcellated in 90 cortical and sub-cortical regions with the automated anatomical labeling (AAL) atlas 39]. Further, we included heterogeneous time delays based in the spatial location of brain regions defined by AAL parcellation 39 (Fig 1B). Connections between nodes are made by pyramidal neurons, considering that long-range projections are mainly excitatory 40.41]. Using the firing rates of each node as inputs to a generalized hemodynamic model [42], we obtained fMRI-BOLD signals from which we calculated integration and segregation of the resulting functional connectivity matrices.

Following Shine et al. [18, we modeled the influence of the cholinergic and noradrenergic systems through the manipulation of the response and filter gain, respectively (Fig 1C). The principal difference in our approach is that we split the response gain in excitatory gain (long-range pyramidal to pyramidal coupling), $\alpha$, and inhibitory gain (local inhibitory to excitatory interneurons coupling), $\beta$. While the excitatory gain boosts pyramidal neurons output, the inhibitory gain reduces the local 


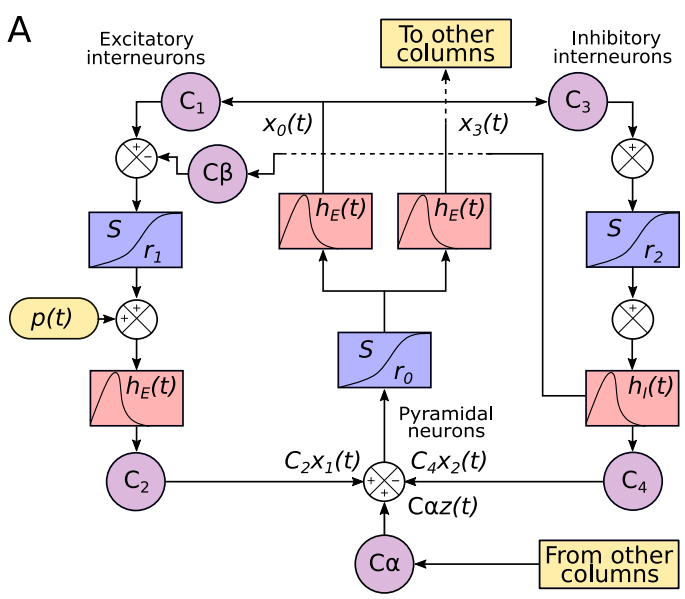

B

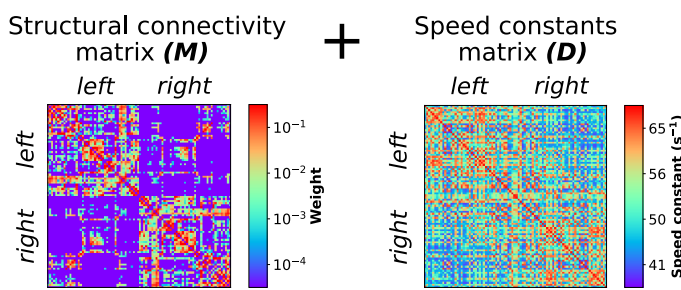

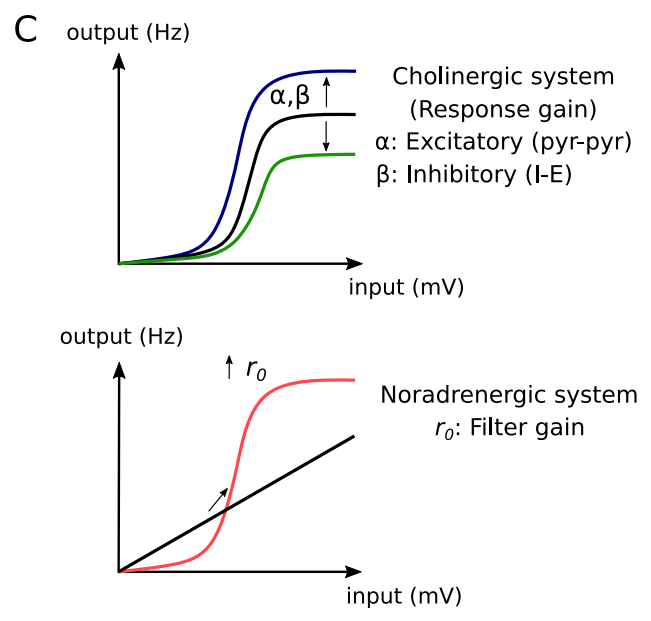

Whole-brain neural mass model

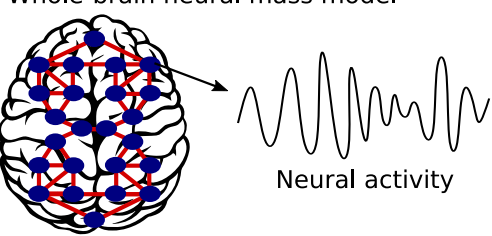

Fig 1. Whole-brain neural mass model.

A) The Jansen \& Rit model is constituted by a population of pyramidal neurons with excitatory and inhibitory feedback mediated by interneurons (INs). Each population is connected by a series of constants $C_{i}$. The outputs are transformed from average pulse density to average postsynaptic membrane potential by an excitatory (inhibitory) impulse response function $h_{E}(t)\left(h_{I}(t)\right)$. Then, a sigmoid function $S$ performs the inverse operation. Pyramidal neurons project to distant cortical columns, and receive both uncorrelated Gaussian-distributed inputs $p(t)$ and inputs from other cortical columns $z(t)$. Neuromodulation is constituted by the excitatory gain $\alpha$, which scales $z(t)$, inhibitory gain $\beta$, which increases the inhibitory input to excitatory INs, and filter gain, $r_{0}$, which modifies the slope of the sigmoid function in pyramidal neurons. B) In the whole-brain model, each node represents a cortical column, whose dynamics is ruled by the Jansen \& Rit equations. Nodes are connected through a structural connectivity matrix, $M$, and a speed constants matrix, $D$. C) Neuromodulation modifies the input (average postsynaptic membrane potential) to output (average pulse density) sigmoid function. The cholinergic system has a multiplicative effect on the sigmoid function. $\alpha$ amplifies the response of pyramidal neurons to other columns' input, while $\beta$ amplifies the effect of inhibitory INs to excitatory INs. On the other hand, the noradrenergic system increments the responsivity of pyramidal neurons to relevant stimuli respect to noise, as a filter, by increasing the slope $r_{0}$ of their sigmoid function.

excitatory feedback from interneurons. Finally, the filter gain $r_{0}$ modifies the sigmoid function slope of pyramidal neurons, increasing its responsivity to relevant stimuli and boosting signal-to-noise ratio. Here, we studied the combined effect of the three gain mechanisms to understand how neuromodulatory systems shape the global neuronal dynamics in two different timescales: EEG-like and BOLD-like signals. Our hypothesis is that the inhibitory gain will play a significant role in increasing the likelihood of integration. 


\section{Inhibitory gain facilitates neuronal coordination}

We first studied the combined influence of the excitatory and inhibitory response gains, by fixing $r_{0}=0.56 \mathrm{mV}^{-1}$ and then simulating neuronal activity at different combinations of $\alpha \in[0,1]$ and $\beta \in[0,0.5]$. Then, we measured the degree of synchrony of the EEG-like signals using the averaged Kuramoto order parameter $\bar{R}[43$. In addition, we analyzed the graph properties of the static (time-averaged) functional connectivity (sFC) matrices, obtained from the pairwise Pearson's correlations of BOLD-like signals. Namely, we calculated the global efficiency $E^{w}$, a measure of integration defined as the inverse of the characteristic path length [4], and modularity $Q^{w}$, a measure of segregation based on the detection of network communities or modules 44. High values of $E^{w}$ represent an efficient coordination between all pairs of nodes in the network, a signature of integration. In contrast, a high modularity $Q^{w}$ is associated to segregation and viceversa 44]. Finally, we measured the mean participation coefficient $P C^{w}$, an integration metric that quantifies the between-modules connectivity, and the transitivity $T^{w}$, which accounts for segregation counting triangular motifs 44 .

Fig 2A shows that functional integration $\left(E^{w}\right)$ and neuronal synchronization $(\bar{R})$ are maximized in an intermediate region of the $(\alpha, \beta)$ parameter space. Also, coordination is accompanied by a decrease in the mean oscillatory frequency, $\omega$, which falls within the EEG-Theta range $(4-8 \mathrm{~Hz})$, and a decrease in the segregation $\left(Q^{w}\right)$. The system undergoes a sharp transition crossing a critical boundary both in EEG and BOLD timescales. This clear delimitation of states is a feature of criticality [45]. The transitions between different regimes are better appreciated in Fig 2B where we show a $1-\mathrm{D}$ sweep of $\alpha$ at $\beta=0.25$. Dashed lines at $\alpha=0.23$ and $\alpha=0.8$ correspond to points in the parameter space where drastic changes in dynamic properties of the network occur. Both $E^{w}$ and $\bar{R}$ follow an inverted-U relationship with excitatory neuromodulation, recovering a known result in this field 19 . Also, $Q^{w}$ peaks higher at the right critical boundary (dashed lines), supporting the hypothesis that further increases of the excitatory gain, mediated by the cholinergic system, promote segregation [10]. A 1-D sweep of $\beta$ at $\alpha=0.5($ Fig 2C $)$, shows an increase in synchronization and integration crossing the critical point at $\beta=0.1$. The aforementioned results are similar for the mean $P C^{w}$ and $T^{w}$, as shown in S1 Fig.

The modulation of the inhibitory gain $(\beta)$ shows a compelling effect on the integration and segregation of the whole network. This could be due to the reduction of excitatory feedback only, or a more specific effect of the connection from inhibitory to excitatory interneurons. In the first case, we expect a similar effect by reducing the $C_{1}$ parameter (see Fig 1A because this also reduces the excitatory feedback loop of the cortical columns. As shown in S2 Fig this is only partially the case. The reduction of the $C_{1}$ connection weight -in the absence of the inhibitory-to-excitatory interneuron connection- enables the network to reach integration but in a smaller region of the parameter space and to a lower extent than the inhibitory modulation that we introduced in our model. This highlights the role of specific intra-columnar inhibitory feedback connections in shaping the network behavior, and justifies our modification of the model as an homeostatic mechanism (see Discussion).

The reduction of the average oscillatory frequency could be an effect of the time delays incorporated in the model, as suggested by Nordenfelt et al. [46] and Lea-Carnall et al. 47]. We found the mean $\omega$ to be a function of the average speed constant $\langle D\rangle$ (not shown), suggesting that whole-brain integration goes together with a reduction in oscillatory frequency, as a consequence of the time delays between cortical columns.

At the functional level, integration is characterized by the increase of inter-modular connectivity. To observe in detail how each gain mechanism produces integrated or segregated states, we show some BOLD-like signals and their respective sFCs matrices 

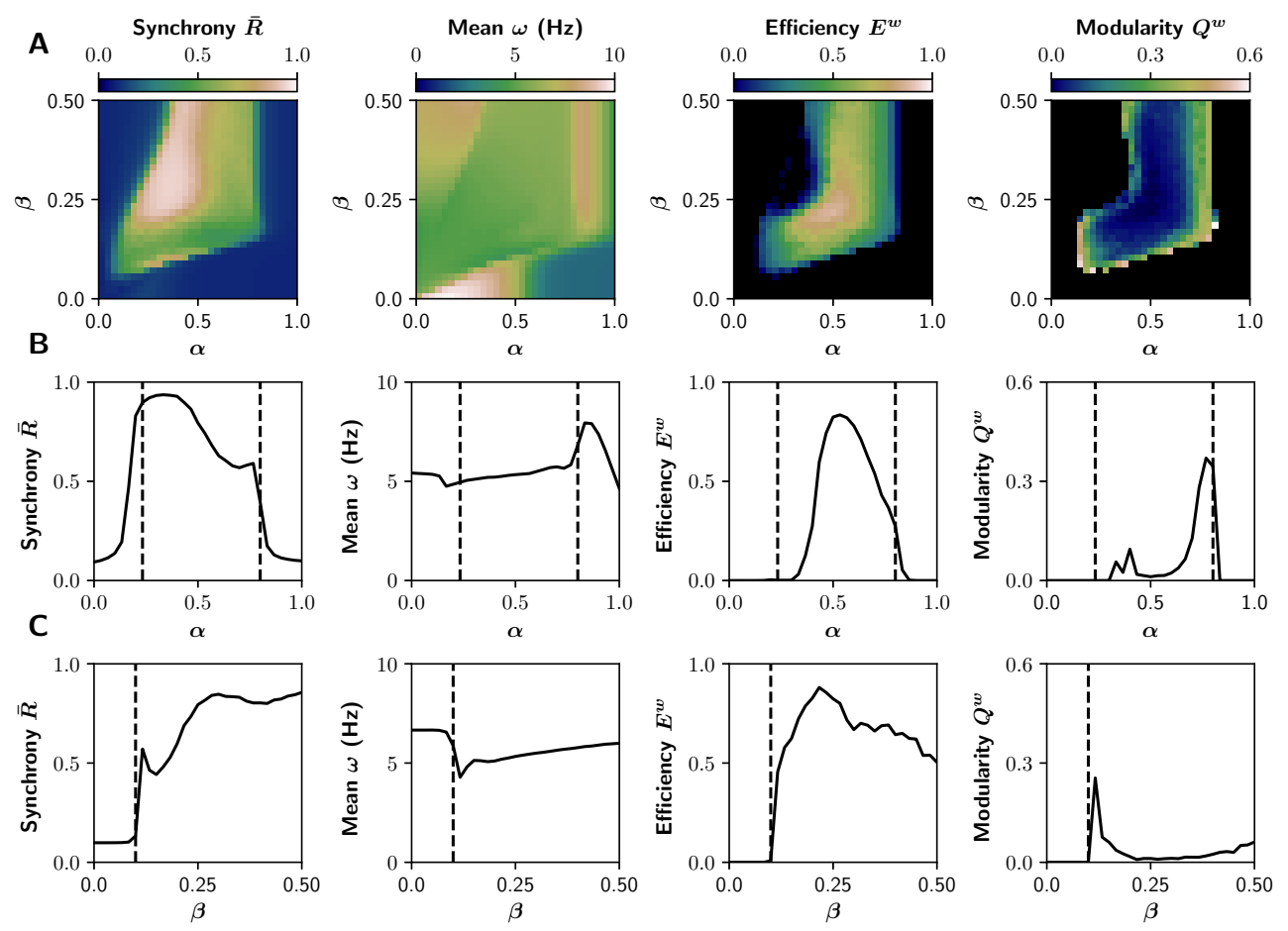

Fig 2. Signal and network features in the $(\alpha, \beta)$ parameter space.

A) Average phase synchrony $\bar{R}$, mean oscillatory frequency $\omega$ of EEG-like signals, global efficiency $E^{w}$ (integration) and modularity $Q^{w}$ (segregation) of the graphs derived from the sFCs of the BOLD-like signals. B) Transitions through critical boundaries in the direction of $\alpha$ axis, for a fixed $\beta=0.25$. Transition points are represented by black dashed lines at $\alpha=0.23$ and $\alpha=0.8$. C) Transitions in the direction of $\beta$ axis, for a fixed $\alpha=0.5$, with a critical point at $\beta=0.1$.

in Fig 3. We chose five tuples of values of $(\alpha, \beta)$, marked with the red circles in the Fig $3 \mathrm{~A}$. We observe that functional integration is maximal in the middle $(\alpha=0.5, \beta=0.25)$, and segregation is promoted far away from this point $(\alpha=0.25, \beta=0.125$, and $\alpha=0.75, \beta=0.375)$. In the extreme cases $(\alpha=0, \beta=0$, and $\alpha=1, \beta=0.5)$ there is neither integration nor segregation.

\section{Inhibitory gain allows the noradrenaline-mediated integration}

The inclusion of the inhibitory gain in the model has the capability of producing novel predictions on the basis of a biophysically plausible mechanism. To validate this model and its results, it should also reproduce the results of the current neuromodulatory paradigm proposed by Shine 10,18 . We characterized the relationship between neuromodulation and integration in the $\left(\alpha, r_{0}\right)$ parameter space, with $\alpha \in[0,1]$ and $r_{0} \in[0,1]$ while leaving $\beta$ fixed at 0 or 0.4 (without and with inhibitory gain, respectively). The results for $\beta=0$ (Fig 4A) show neither integration nor synchronization in the entire parameter space. On the other hand, the observations of Shine et al. 18 are fully reproduced with $\beta=0.4($ Fig 4B $)$, supporting the fact that inhibitory gain is essential to produce neuronal coordination. Similar results hold for the mean $P C^{w}$ and $T^{w}$, as shown in S3 Fig,

As observed previously in Fig 2 , a critical boundary delimits asynchronous and synchronous states in the $\left(\alpha, r_{0}\right)$ parameter space, a signature of criticality [45]. This 

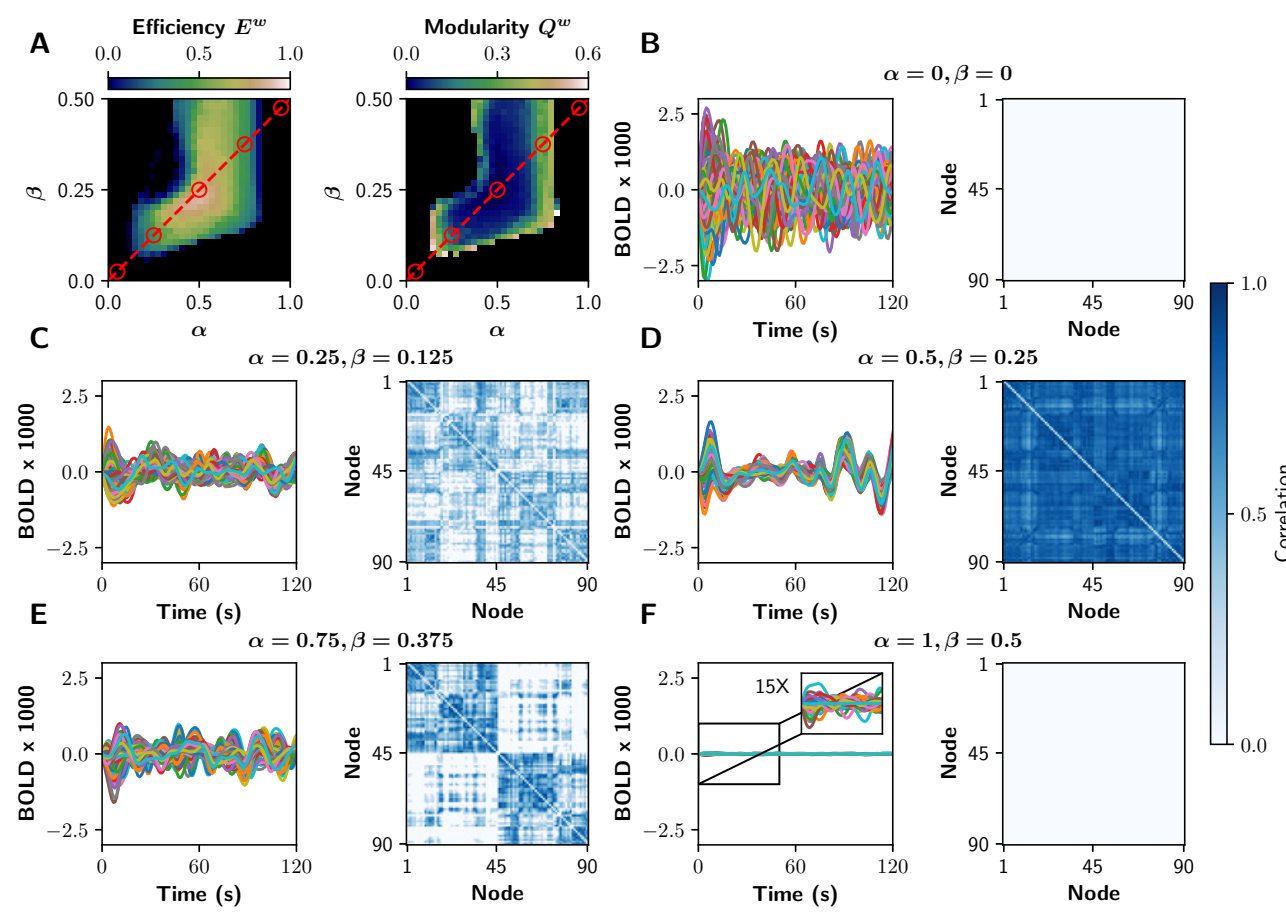

Fig 3. fMRI-like sFCs at different values of $\alpha$ and $\beta$.

A) The red circles represent pairs of $(\alpha, \beta)$ values in which different integration/segregation profiles can be observed. B-F) BOLD-like signals, and their respective sFCs matrices, for the $(\alpha, \beta)$ shown in A. We shown the first $120 \mathrm{~s}$ of BOLD-like signals, while sFCs matrices were built with the full-length time series (600 s).

behavior was reported before in the original neuromodulatory framework of Shine et al. 18. A 1-D sweep of $\alpha$ at $r_{0}=1 \mathrm{mV}^{-1}$ shows a sharp transition (Fig 4C). Mean phase synchrony $\bar{R}$ increments alongside global efficiency $E^{w}$, changes that go along with a decrease of the average frequency $\omega$, and a reduction of modularity $Q^{w}$. However, further increments of $\alpha$ produce network desynchronization. On the other hand, a 1-D sweep of $r_{0}$ at $\alpha=0.6$ (Fig 4D produces similar observations, but just one boundary is visible. These results support the hypothesis that noradrenergic and cholinergic systems, combined, promote integration following an inverted-U relationship [4,11, 18, but only if the inhibitory gain is included.

In the whole-brain model, the cholinergic system exerts its effect by changing both $\alpha$ and $\beta$ parameters. Under this assumption, a logical consequence of the cholinergic neuromodulation is the possibility of simultaneously increase/decrease of $\alpha$ and $\beta$. For that reason, we repeated the analysis previously performed in the $\left(\alpha, r_{0}\right)$ parameter space, but this time we changed $\beta$ alongside $\alpha$ following the relationship $\beta=0.35 \alpha$. The results are shown in S4 Fig. They are similar to those in Fig 4 , but this time the relationship between $r_{0}$ and $E^{w}$ is no longer a sigmoid-like function, rather it follows an inverted-U relationship. The simultaneously modulation of $\alpha$ and $\beta$ has a consequence to the excitability levels of pyramidal cells. For example, when $\alpha \sim 0.5$ the maximum integration could be achieved at lowers levels of $r_{0}$. Increasing $r_{0}$ further produces an over-excitation of pyramidal cells. In consequence, for $\alpha=0.5$, the optimal $r_{0}$ value for maximizing integration in S4 Fig ( with $\beta=0.35 \alpha=0.175$ ) would be lower in comparison with Fig 4 (with a fixed $\beta=0.4$ ). Thus, the simultaneous modulation of 

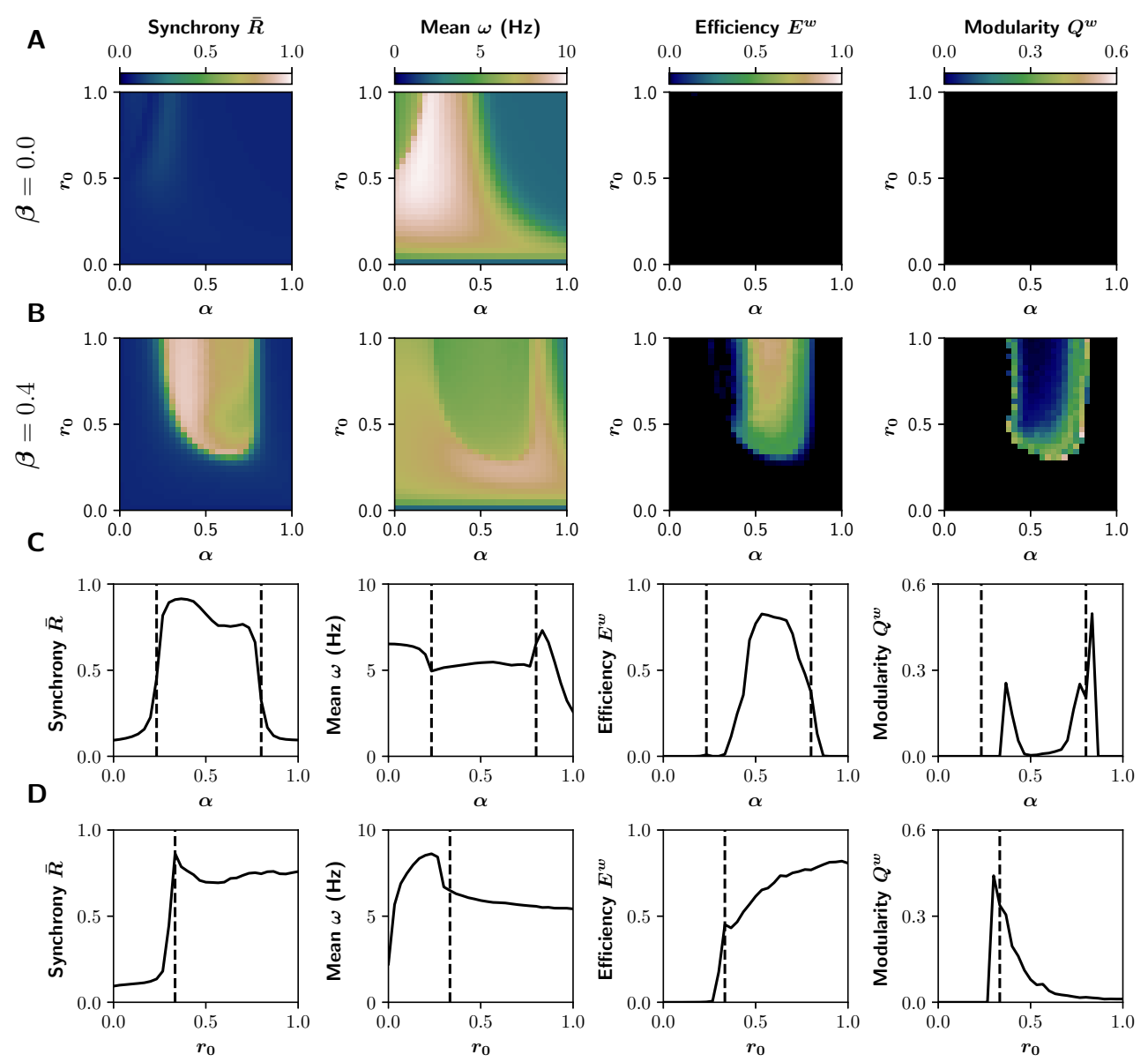

Fig 4. Signal and network features in the $\left(\alpha, r_{0}\right)$ parameter space.

A-B) Average phase synchrony $\bar{R}$ and mean oscillatory frequency $\omega$, and global efficiency $E^{w}$ (integration) and modularity $Q^{w}$ (segregation) of the graphs derived from the sFCs of the BOLD-like signals, for $\mathbf{A}$ ) $\beta=0$ (no action of the inhibitory gain) and B) $\beta=0.4$. C) Transitions through the critical boundary in the direction of $\alpha$ axis, with a fixed $r_{0}=1 \mathrm{mV}^{-1}$ and $\beta=0.4$. Critical transition points represented by black dashed lines at $\alpha=0.23$ and $\alpha=0.8$. D) Transitions in the direction of $r_{0}$ axis, for a fixed $\alpha=0.6$, with a critical point at $r_{0}=0.33 \mathrm{mV}^{-1}$ and $\beta=0.4$.

both excitatory and inhibitory circuits in a whole brain model, allowed to recover an empirical feature that was not previously reproduced 19 .

\section{Signal-to-noise ratio and regularity matches with neuronal coordination}

Previous experimental and theoretical works 10, 11,22 suggest that neuromodulatory systems increase the signal-to-noise ratio, allowing neuronal populations to be sensitive to local or distant populations to a greater extent than noise. To test that, we measured the signal-to noise-ratio (SNR) using the power spectral density (PSD) function of each signal (see Methods) and report the average value over all nodes. Additionally, we computed the regularity index [48], as a measure of signal periodicity. This metric is defined as the second absolute peak of the autocorrelation function and is bounded 

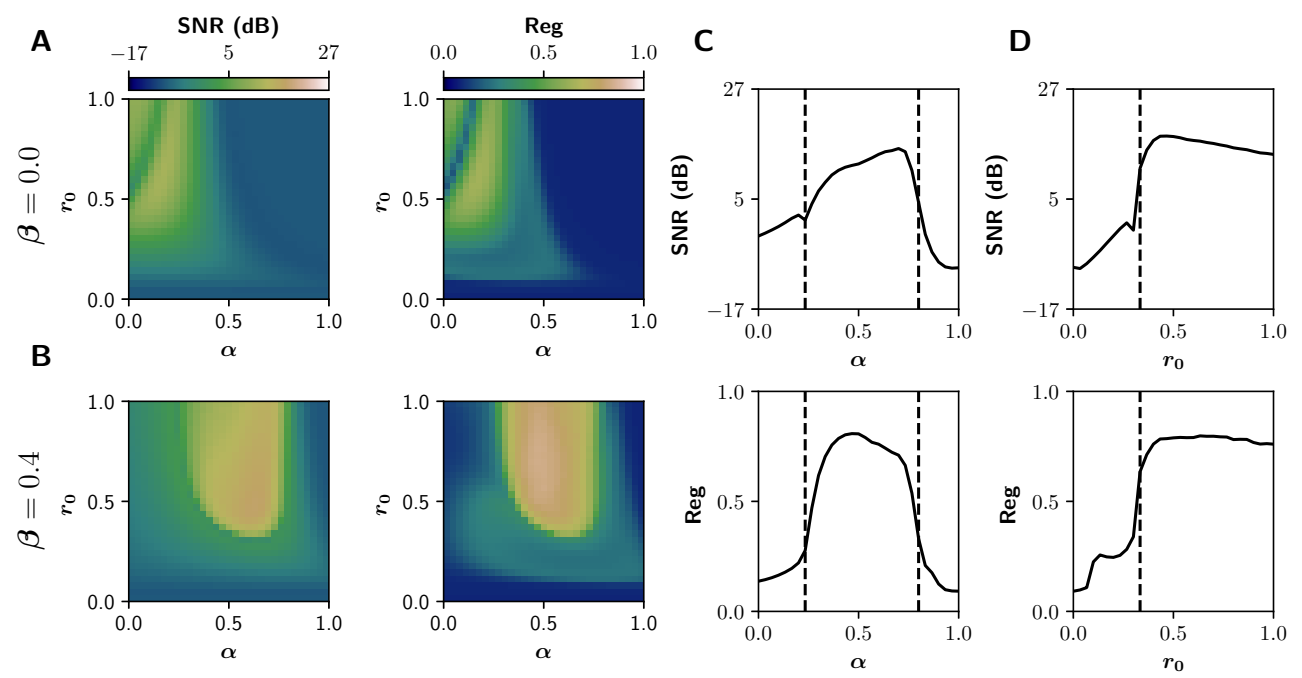

Fig 5. Signal-to-noise ratio (SNR) and regularity (Reg), for the EEG-like signals, in the $\left(\alpha, r_{0}\right)$ parameter space.

A) No action of inhibitory gain $(\beta=0)$. B) SNR and regularity matches with functional integration for $\beta=0.4$. C) Transitions through the critical boundary in the direction of $\alpha$ axis, with a fixed $r_{0}=1 \mathrm{mV}^{-1}$. Critical points represented by black dashed lines at $\alpha=0.23$ and $\alpha=0.8$. D) Transitions in the direction of $r_{0}$ axis, for a fixed $\alpha=0.6$, with a critical point at $r_{0}=0.33 \mathrm{mV}^{-1}$.

between 0 and 1 , with 0 for purely chaotic or noisy signals, and 1 for perfectly periodic signals. We report the average overall nodes.

Both SNR and regularity match the region of neuronal synchronization and integration (Fig 5), supporting the idea that neuromodulatory systems promote integration by increasing SNR. These results are not possible without the action of inhibitory gain $(\beta=0$, Fig $5 \mathrm{~A})$. As mentioned before, in conditions of reduced excitatory feedback, the excitatory response gain increases the output of pyramidal neurons above noise and, consequently, boosts SNR. Then, the increase of filter gain raises the sensitivity of neuronal populations to inter-columnar stimulation.

The increment in regularity suggests that a partial reduction of signal stochasticity is necessary for the whole-brain functional integration, a result supported by the idea that ordered in-phase synchronization is an essential and cost-efficient way to coordinate the brain activity 49 . Interestingly, regularity is near 0.5 at the critical boundary (Fig 5D), placing the signals between order and disorder, in which high amplitude synchronized oscillations alternate with noisy unsynchronized signals.

\section{Dynamical richness peaks near the critical boundary}

We tested the hypothesis that network stability is higher in integrated states, and neuronal variability peaks near the critical boundaries. In the faster timescale (EEG), we computed the metastability $\chi_{R}$ of bandpass filtered EEG-like signals, using a metric defined as the variance of the Kuramoto order parameter [50]. Values closer to 0 are expected for completely asynchronous or completely synchronous activity, and greater than 0 when the neuronal activity exhibits periods of high and low synchronization. In the slowest timescale, we performed a Functional Connectivity Dynamics (FCD) analysis 8,9 over the BOLD-like signals, using the sliding windows approach depicted in the Fig $6 \mathrm{~A}-\mathrm{C}$ [51]. The resulting time vs time FCD matrix captures the concurrence 
A

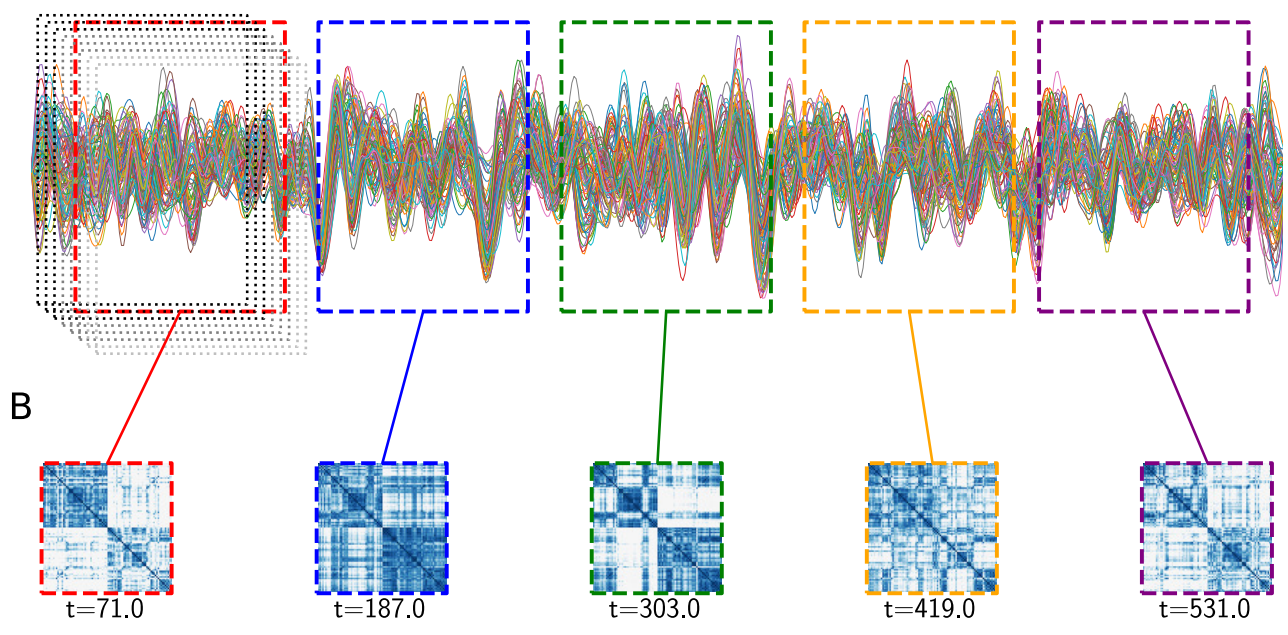

C

D

FCDs through the $\alpha$ boundary
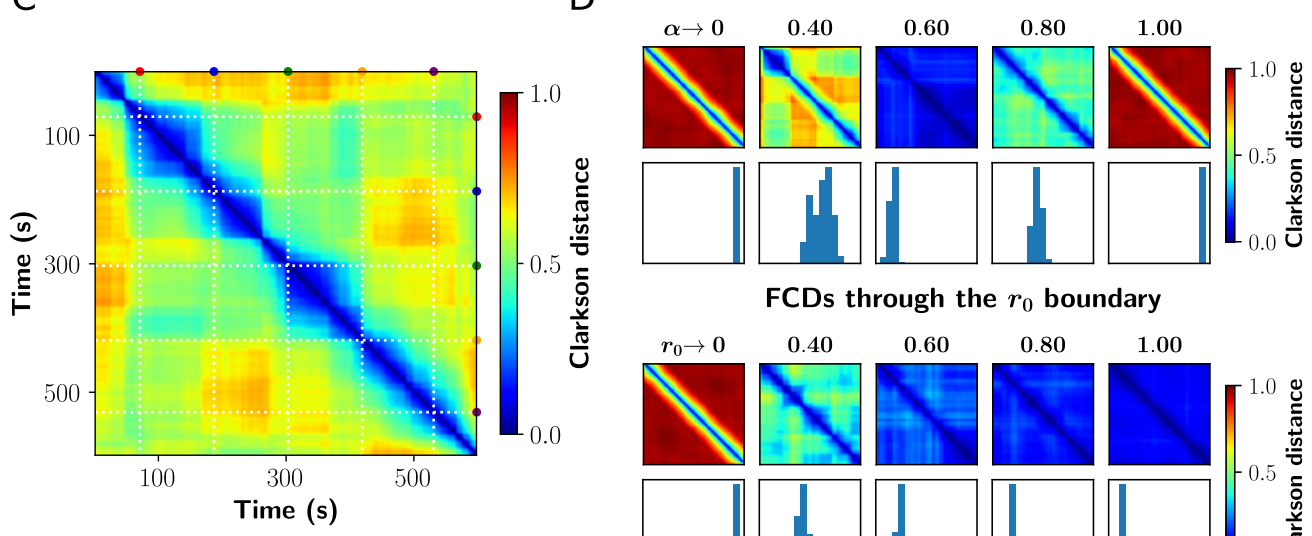

FCDs through the $r_{0}$ boundary

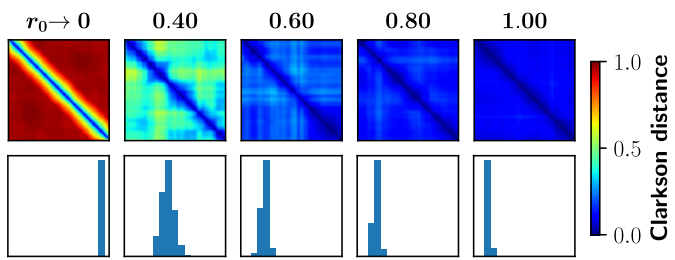

Fig 6. Analysis of Functional Connectivity Dynamics.

A) Sample fMRI-BOLD time series showing the fixed length and overlapping time windows at the begginging. In color, the time windows corresponding to the FCs shown in B. B) FCs matrices obtained in the colored time windows. C) Functional

Connectivity Dynamics (FCD) matrix, where all the FCs obtained were vectorized and then compared against each other using a vector-based distance (Clarkson distance). D) FCDs matrices through the critical boundary, in both $\alpha$ and $r_{0}$ direction. Below each FCD, a histogram of its upper triangular values is shown. The variance of these values constitutes a measure of multistability.

of FC patterns, visualized as square blocks. We computed the variance of the FCD, $\operatorname{var}(\mathrm{FCD})$, as a multistability index [51], where values greater than 0 indicate the switching between different FC patterns. Both metastability and multistability are measures of dynamical richness. Additionally, we calculated the FCD speed $d_{t y p}$ as described by Battaglia et al. [52, which captures how fast the FC patterns fluctuate over time. Values closer to 1 indicate a recurrent change of diverse FC patterns, and closer to 0 the concurrence of stable and similar states over time.

In Fig 6D we show a set of FCD matrices obtained at different values of $\alpha$ and $r_{0}$, together with histograms of their off-diagonal values. Red FCD matrices (with high 
values) correspond to incoherent states, as the $\mathrm{FC}$ continuously evolve in time. On the other hand, a blue FCD matrix (with low values) indicates a fixed FC throughout the simulation. Multistability is higher for green/yellow patchy matrices, because this indicates FC patterns that change and also repeat over time. As can be inferred observing the FCD distributions, the variance of the values in the histograms $(\operatorname{var}(\mathrm{FCD}))$ can be used as a measure of multistability [51].

Fig 7 shows how metastability, multistability (FCD variance) and FCD speed change in the whole $\left(\alpha, r_{0}\right)$ space. At low levels of both $\alpha$ and $r_{0}$, the neuronal activity is constituted mainly by noisy asynchronous signals, conditions associated to low (near 0 ) values of $\chi_{R}$ and $\operatorname{var}\left(\mathrm{FCD}\right.$ ), and with a high $d_{t y p}$ (all FC patterns differ from each other, as expected for noise-driven signals) (Fig 7A). In the other extreme, for $r_{0}>0.5$ $\mathrm{mV}^{-1}$ and $\alpha \in[0.4,0.6]$, values that correspond to the integrated states, $\operatorname{var}(\mathrm{FCD})$ is also small and $d_{t y p}$ falls close to 0 . In consequence, integrated states are more stable and less susceptible to network reconfiguration over time. In contrast, both $\chi_{R}$ and $\operatorname{var}(\mathrm{FCD})$ peak near the critical boundary, through the direction of $\alpha$ and $r_{0}$ axes $($ Fig 7B-C . Moreover, crossing the boundary is associated with a continuous decrease of $d_{t y p}$ : the emerging integration mediated by gain mechanisms is associated with more stable FCs patterns over time. These observations are in agreement with experimental results using fMRI, in which the network variability increases in resting-state, and decreases with network integration during cognitive tasks [3].

\section{Discussion}

Based on previous experimental findings $20,23,26,27$, we hypothesized that the cholinergic neuromodulation of the inhibitory interneurons (that suppresses the local excitatory feedback to pyramidal neurons) facilitates functional integration. Our results confirm this hypothesis in the context of a whole-brain neural mass model, and hold simultaneously for EEG-like and fMRI-like signals obtained considering different time scales. The main novelty of our work is the inclusion of the inhibitory gain (the parameter $\beta$ ) in the model and the characterization of its influence in the functional integration.

Several clues suggest that the model we proposed is in the right track. First, our results reproduce the inverted-U relationship between cholinergic neuromodulation and integration, reported experimentally [11,19] and reproduced in a similar whole-brain model [10,18]. Further, functional integration matches with an increase of SNR, a common effect attributed to neuromodulatory systems [11,22]. The reduction of the variability of the signals (higher regularity) found in our model has also been reported experimentally in resting-state fMRI, related to an increase in functional

connectivity [53]; moreover the SNR, signal variability, and functional connectivity have been shown to be influenced by neuromodulatory systems [54]. Then, the reduction in oscillatory frequency observed in the model -which falls within the Theta range of EEG spectrum- has also been perceived in several cognitive tasks [55, 56, and is a

consequence of the time delays as suggested by other computational studies [46, 47. Finally, the model is capable to reproduce the previous results of Shine et al. [18]: the increase in phase synchronization and functional integration with neuromodulation, the reduction of the time-resolved topological variability with integration (in our case captured by the variance of the FCD), and the inverted-U relationship of the excitatory gain $\alpha$ with $E^{w}$ and mean $P C^{w}$.

Our main result is that the action of the cholinergic system on both, the excitation of pyramidal neurons and the intra-columnar inhibitory feedback, is needed to shift from a regime of unsynchronized activity, towards a regime of coherent activity (integrated). The inclusion of the additional inhibitory-to-excitatory interneuron 

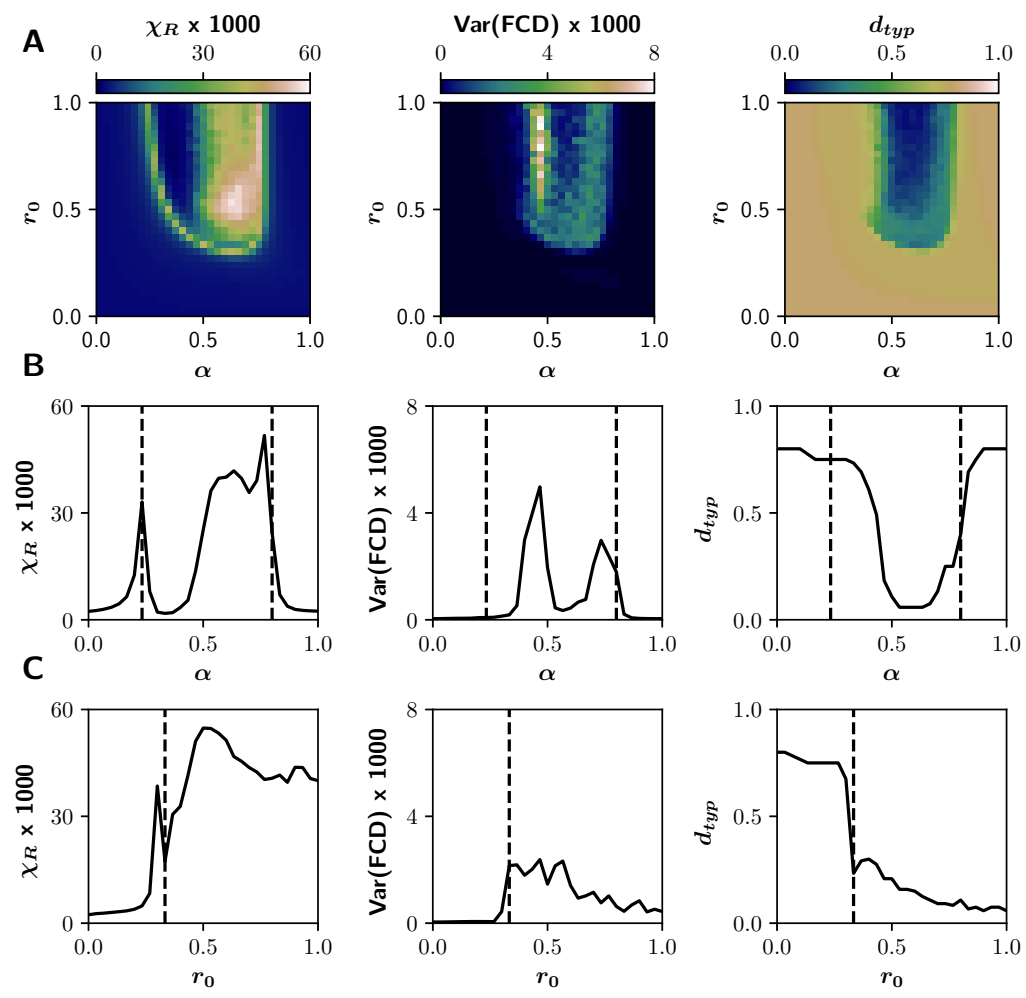

Fig 7. Dynamical features of the system in the $\left(\alpha, r_{0}\right)$ parameter space. A) Metastability $\chi_{R}$ calculated as the variance of the Kuramoto order parameter (EEG-like signals), multistability $\operatorname{var}(\mathrm{FCD})$ and typical FCD speed $d_{\text {typ }}$ measured from the Functional Connectivity Dynamics analysis (BOLD-like signals). While $\chi_{R}$ and $\operatorname{var}(\mathrm{FCD})$ peak on the critical boundary, $d_{t y p}$ decreases crossing the dashed lines (critical points) and reaches its minimum values in integrated states. B) Transitions through the critical boundary in the direction of $\alpha$ axis, with a fixed $r_{0}=1 \mathrm{mV}^{-1}$. Critical points represented by black dashed lines at $\alpha=0.23$ and $\alpha=0.8$. C) Transitions in the direction of $r_{0}$ axis, for a fixed $\alpha=0.6$, with a critical point at $r_{0}=0.33 \mathrm{mV}^{-1}$.

connection, and its modulation through the parameter $\beta$, facilitates the emergence of coordinated regimens of activity (compare the cases for $\beta=0$ and $\beta=0.4$ of Fig 4 ). In our model, the inhibitory gain $\beta$ has a double effect on the dynamic of the network. On one hand, it increases the relative magnitude of the inter-columnar afferences (compared to the internal excitatory loop), and on the other hand, it provides a simple dynamical mechanism to homeostatically preserve the excitation/inhibition (E/I) balance at the node level. In fact, this balance may be considered a determinant element in the interplay between integration and segregation [31. Interestingly, this mechanism maximizes the functional integration in a better way than a direct decrease of the internal excitatory feedback, as we evidenced with the reduction of $C_{1}$ (see Fig 2 and S2 Fig for a comparison). Our model suggests that the additional inhibitory loop, modulated by the inhibitory gain $\beta$, constitutes an optimal solution to the dynamical control of cortical column activity.

The inhibition-mediated control of the E/I balance has been implemented in others networks models of brain activity [57]. At the whole-brain level, Deco et al. [58] employed a feedback inhibitory control (FIC) to preserve the E/I balance in a mean-field network connected with a human connectome, producing the best fit 
between the simulated and empirical sFCs matrices. Also, FIC improved the information capacity of the global network and enhanced its dynamical repertoire constituting a biophysical plausible mechanism to reproduce the macro-scale features of human brain dynamics 58. In our model, we implemented instead a disynaptic inhibitory control [36 37 that, in spite of leading to similar results, can present additional advantages. The inhibition included in the model (modulated by the inhibitory gain $\beta$ ) influences the output of pyramidal neurons when excitability is high 36 . Because the two populations of interneurons receive excitatory inputs from pyramidal cells, an increase in pyramidal excitability triggers both the feedback excitation loop and its dampening by the inhibitory interneurons. Conversely, when the pyramidal excitability decreases, the effect of the inhibitory loop between interneurons is low, and the excitatory loop can rise the excitability of pyramidal cells. This constitutes and effective mechanism to maintain an optimal E/I balance. In contrast, a direct reduction of the excitatory feedback (e.g., decreasing $C_{1}$, S2 Fig), produces no compensation when pyramidal excitability is low.

Another contribution of our work is that we propose an explicit mechanism of interaction between meso-scale properties of brain activity, such as the local inhibition, with the global increase in functional integration. While the cholinergic system increases the response of pyramidal neurons to external afferences (e.g., inputs from other brain areas) through nicotinic and muscarinic receptors [21], the increase in intra-cortical inhibition could be mediated mainly by nicotinic receptors 20.23. At the meso-scale level, nicotinic receptors can increment interneurons activity in a similar way than the inhibitory gain $\beta 20,22,23$. However, there is little knowledge about the specific effects of the cholinergic system at the whole-brain level. In that line, an increase in the local and global efficiency in resting-state fMRI has been reported after the administration of nicotine [59], and some nicotinic agonists have pro-cognitive effects as well in health and disease 60 . Considering the relationship between function integration and cognition 2 $2,3,10$, our model suggests that the possible pro-cognitive effects associated with the cholinergic system are related to a selective increase in the excitability of excitatory and inhibitory neural populations within brain areas. Thus, our computational approach -in the same spirit as Wylie et al. [59]- links the meso-scale consequences of inhibitory interneurons neuromodulation with the functional network topology features, at the whole-brain level.

The inverted-U relationship between neuromodulation and integration that we found in our model is a consequence of the dynamics of individual cortical columns. At the node level, the parameters we used in the Jansen \& Rit model put the model near a Hopf supercritical bifurcation [61. When $\alpha$ and $r_{0}$ are low, the node dynamics is defined by a stable focus (a fixed point with non-monotonic convergence), and thus pyramidal outputs consist of low amplitude noisy signals. Increasing both parameters causes the bifurcation into an unstable focus within a limit cycle, with high amplitude oscillations. Increasing $\alpha$ further produces a new bifurcation (a stable focus) and the limit cycle disappear. The inhibitory gain $\beta$ constitutes a mechanism to keep the model working between the two bifurcations points; it preserves the E/I balance as an inhibitory control loop. At first, and likewise Shine et al. [18, we did not find an inverted-U relationship between the filter gain -modulated by the noradrenergic systemand integration. In the brain, the analogous of our parameters $\alpha$ and $\beta$ are modulated -in parallel- by the cholinergic system, and noradrenaline has an additional effect in increasing excitability [54, thus it is possible that the inverted-U relationship observed between neuromodulation and integration is a consequence of a complex interaction between neuromodulatory systems [18]. We confirmed this changing, in parallel, both $\alpha$ and $\beta$ parameters (see $\mathrm{S} 4 \mathrm{Fig}$ ). The simultaneously modulation reproduced, at least partially, the inverted-U relationship between the noradrenergic system -in our model 
represented by the filter gain $r_{0}$ - and functional integration measured as the global efficiency $E^{w}$. However, as the cholinergic effects on excitatory and inhibitory systems are mediated by different receptors with different expression patterns, there can be many ways by which the integration/segregation balance is controlled by neuromodulatory systems.

Near the critical boundary, in the $\left(\alpha, r_{0}\right)$ parameter space, we observe an increase in the metastability and multistability, a decrease in the FCD speed, and the FCD analysis suggests an increment in dynamical richness, a consequence of the fact that each node is poised close to a Hopf supercritical bifurcation [5]. It has been proposed that at rest brain activity operates near a bifurcation point, where segregated (uncoordinated) and integrated (coordinated) regimes alternate in time [5, 8, 9. In our model, the neuromodulation facilitates the transitions from "resting-state" conditions, near the critical points, to more integrated regimes. Furthermore, integrated regimes become more stable in time, as can be noted with the decrease in $d_{t y p}$. Analogously, time-resolved functional connectivity analysis, in task-related fMRI recordings, indicates a decrease in the topological variability in subjects performing an N-back task; the extent of integration acts as a predictor of individual performance and response times 3]. Our model reproduces the variability observed in "resting-state" conditions, near the critical points in the parameter space, and the switching to integrated regimes with neuromodulation. Moreover, the effect of the inhibitory gain in preserving the E/I balance can play an important role in sustaining the metastability and multistability at rest. Indeed, the E/I balance is a key element to support criticality 62 and to modulate the integration and segregation features of the brain [45].

There is a lot of room for further progress starting from this work. Future research may consider the addition of neuromodulatory maps 38, 63] in order to take into account the heterogeneous expression of the receptors, or explore models that can reproduce the effect of other neuromodulatory systems [64 and their dynamics 65]. Other interneurons subtypes and their modulation could be included - such as fast-spiking inhibitory interneurons- to account for the faster EEG features of brain activity [66]. Additionally, the graph theoretical analysis used here only consider pairwise interactions, neglecting high-order effects that may contain important information about high dimensional functional brain interactions.

Information-theoretical [67,68] and algebraic topological approaches [57,69, 70] may provide complementary insights of high-order interdependencies in the brain.

In summary, our results extend the neuromodulatory framework proposed by Shine [10], with the inclusion of the cholinergic neuromodulation of inhibitory interneurons. Importantly, we describe the homeostatic effect of this local inhibitory feedback loop, a meso-scale mechanism that interacts with macro-scale behavior and enables functional integration. Our findings shed light on a better understanding of neurophysiological mechanisms involved in the functional integration and segregation of the human brain activity. This line of research may have plentiful of scientific and clinical implications, as a vast body of evidence suggest that functional integration and segregation may be altered in neuropsychiatric disorders $31,71,73$. Therefore, understanding the neuromodulatory mechanisms that underlie the imbalances of integration and segregation may lead to future progress in pharmacological treatments.

\section{Materials and methods}

\section{Whole-Brain Neural Mass Model}

To simulate neuronal activity we used a reparameterized version of the Jansen \& Rit neural mass model [32]. In this model, a cortical column consists of a population of 
pyramidal neurons with projections to other two populations: excitatory and inhibitory interneurons, which project back to the pyramidal population. Additionally, the pyramidal neurons receive an external stimulus $p(t)$, whose values are taken from a Gaussian distribution with mean $\mu=2 \mathrm{~Hz}$ and standard deviation $\sigma=2.25$. Different values of $\sigma$ were explored and our results are mostly consistent for $1.5<\sigma<5$.

The dynamical evolution of the three populations within the cortical column is modeled by two blocks each. The first transforms the average pulse density in average postsynaptic membrane potential (which can be either excitatory or inhibitory) Fig 1A. This block, denominated post synaptic potential (PSP) block, is represented by an impulse response function

$$
h_{E}(t)= \begin{cases}\text { Aate } e^{-a t}, & t \geq 0 \\ 0, & t<0\end{cases}
$$

for the excitatory outputs, and

$$
h_{I}(t)= \begin{cases}B b t e^{-b t}, & t \geq 0 \\ 0, & t<0,\end{cases}
$$

for the inhibitory ones. The constants $A$ and $B$ define the maximum amplitude of the PSPs for the excitatory (EPSPs) and inhibitory (IPSPs) cases respectively, while $a$ and $b$ represent the inverse time constants for the excitatory and inhibitory postsynaptic action potentials, respectively. The second block transforms the postsynaptic membrane potential in average pulse density, and is given by a sigmoid function of the form

$$
S(\nu, r)=\frac{\zeta_{\max }}{1+e^{r(\theta-\nu)}}
$$

with $\zeta_{\max }$ as the maximum firing rate of the neuronal population, $r$ the slope of the sigmoid function, and $\theta$ the half maximal response of the population.

To study the effect of the neuromodulatory systems at the macro-scale level, we included long-range pyramidal-to-pyramidal neurons and short-range inhibitory to excitatory interneurons couplings, to mimic the effects of neuromodulation through the excitatory and inhibitory gain parameters, respectively. This short-range coupling between interneurons, well described at the meso-scale level [36 37], constitutes a modification of the original equations. In the model presented in the Fig 1A, each node $i \in N$, with $N=[1 \ldots n]$ as the set of all nodes of the network, represents a single brain area. The nodes are connected by a structural connectivity matrix $M$ (Fig 1B) This matrix is derived from a human connectome 38 parcellated in $n=90$ cortical and subcortical regions with the automated anatomical labelling (AAL) atlas [39]; the matrix is undirected and takes values between 0 and 1 . Because long-range connections are mainly excitatory 40,41, only links between the pyramidal neurons of a node $i$ with pyramidal neurons of a node $j$ are considered. The model includes a speed constants matrix $D$ for the inter-columnar coupling (Fig 1B). For building the matrix $D$, the distance between regions' centroids defined by the AAL atlas was used. The entries of $D$ decrease exponentially with the distance.

The overall set of equations, for a node $i$, includes the within and between nodes 


$$
\begin{aligned}
\text { activity } & \\
x_{0, i}(t) & =y_{0, i}(t) \\
y_{0, i}(t) & =A a\left[S\left(C_{2} x_{1, i}(t)-C_{4} x_{2, i}(t)+C \alpha z_{i}(t), r_{0}\right)\right]-2 a y_{0, i}(t)-a^{2} x_{0, i}(t) \\
x_{1, i}(t) & =y_{1, i}(t) \\
y_{1, i}(t) & =A a\left[p(t)+S\left(C_{1} x_{0, i}(t)-C \beta x_{2, i}, r_{1}\right)\right]-2 a y_{1, i}(t)-a^{2} x_{1, i}(t) \\
x_{2, i}(t) & =y_{2, i}(t) \\
\dot{y}_{2, i}(t) & =B b\left[S\left(C_{3} x_{0, i}(t), r_{2}\right)\right]-2 b y_{2, i}(t)-b^{2} x_{2, i}(t) \\
x_{3, i j}(t) & =y_{3, i j}(t) \\
y_{3, i j}(t) & =A D_{i j}\left[S\left(C_{2} x_{1, i}(t)-C_{4} x_{2, i}(t)+C \alpha z_{i}(t), r_{0}\right)\right]-2 D_{i j} y_{3, i j}(t)-D_{i j}^{2} x_{3, i j}(t)
\end{aligned}
$$

where $x_{0}, x_{1}, x_{2}$ correspond to the outputs of the PSP blocks of the pyramidal neurons, and excitatory and inhibitory interneurons, respectively, and $x_{3, i j}$ the output from the pyramidal neuron $j$ to the column $i$. Constants $C_{1}, C_{2}, C_{3}$ and $C_{4}$ scale the connectivity between the neural populations (see Fig $1 \mathrm{~A}$ ). We used the original values of Jansen \& Rit [32]: $\zeta_{\max }=5 \mathrm{~Hz}, \theta=6 \mathrm{mV}, r=0.56 \mathrm{mV}^{-1}, a=100 \mathrm{~s}^{-1}, b=50 \mathrm{~s}^{-1}$, $A=3.25 \mathrm{mV}, \bar{B}=22 \mathrm{mV}, C_{1}=C, C_{2}=0.8 C, C_{3}=0.25 C, C_{4}=0.25 C$, and $C=135$.

The overall input to the column $i$ from other cortical columns is given by

$$
z_{i}(t)=\frac{\sum_{j=1, j \neq i}^{n} M_{i j} x_{3, i j}(t)}{\sum_{j=1, j \neq i}^{n} M_{i j}}
$$

The neuronal activity of the column $i$ is the average PSP of pyramidal neurons and characterizes the EEG-like signal in the source space; it is computed as 32

$$
\nu(t)_{i}=C_{2} x_{1, i}(t)-C_{4} x_{2, i}(t)+C \alpha z_{i}(t)
$$

The firing rates of pyramidal neurons $\zeta_{i}(t)=S\left(\nu(t)_{i}, r_{0}\right)$ were used to simulate the fMRI-BOLD recordings. The parameters $\alpha, \beta$ and $r_{0}$ account for the influence of the neuromodulatory systems (Fig 1C), as described in next subsection.

\section{Neuromodulation}

The effects of the cholinergic system were modeled by the parameters $\alpha$ and $\beta$. The parameter $\alpha$ increases the long-rage pyramidal to pyramidal neuron coupling through the $M$ matrix and amplifies the firing rates of the target populations 10 11. The parameter $\beta$ scales the short-range inhibitory to excitatory interneurons coupling, decreasing the recurrent excitation to pyramidal neurons [22]. We refer to $\alpha$ as the excitatory gain, and $\beta$ as the inhibitory gain. In comparison with the current framework proposed by Shine [10, the novelty of our neuromodulatory approach is the inclusion of the inhibitory gain to the model. The effect of the noradrenergic system, designated as filter gain, was simulated controlling the parameter $r_{0}$, which represents the sigmoid function slope of the pyramidal population. This last neuromodulatory system increases the signal-to-noise ratio and inter-columnar connectivity, promoting integration 11.17].

\section{Simulation}

Following Birn et al. 74, we ran simulations to generate the equivalent of $11 \mathrm{~min}$ real-time recordings, discarding the first $60 \mathrm{~s}$. The system of differential equations (1) was solved with the Euler-Maruyama method, using an integration step of $1 \mathrm{~ms}$. We used six random seeds which controlled the initial conditions and the stochasticity of the simulations. We simulated neuronal activity sweeping the parameters $\alpha \in[0,1]$, $\beta \in[0,0.5]$ and $r_{0} \in[0,1]$. All the simulations were implemented in Python and the codes are freely available at https://github.com/vandal-uv/Neuromod2020. 


\section{Simulated fMRI-BOLD Signals}

We used the firing rates $\zeta_{i}(t)$ to simulate BOLD-like signals from a generalized hemodynamic model 42 . An increment in the firing rate $\zeta_{i}(t)$ triggers a vasodilatory response $s_{i}$, producing blood inflow $f_{i}$, changes in the blood volume $v_{i}$ and deoxyhemoglobin content $q_{i}$. The corresponding system of differential equations is

$$
\begin{aligned}
& \dot{s}_{i}(t)=\zeta_{i}(t)-\frac{s_{i}(t)}{\tau_{s}}-\frac{f_{i}(t)-1}{\tau_{f}} \\
& \dot{f}_{i}(t)=s_{i}(t) \\
& \dot{v}_{i}(t)=\frac{f_{i}(t)-v_{i}(t)^{1 / \kappa}}{\tau_{v}} \\
& \dot{q}_{i}(t)=\frac{\frac{f_{i}(t)\left(1-\left(1-E_{0}\right)^{1 / f_{i}(t)}\right)}{E_{0}}-\frac{q_{i}(t) v_{i}(t)^{1 / \kappa}}{v_{i}(t)}}{\tau_{q}},
\end{aligned}
$$

where $\tau_{s}, \tau_{f}, \tau_{v}$ y $\tau_{q}$ represent the time constants for the signal decay, blood inflow, blood volume and deoxyhemoglobin content, respectively. The stiffness constant (resistance of the veins to blood flow) is given by $\kappa$, and the resting-state oxygen extraction rate by $E_{0}$. Finally, the BOLD-like signal of node $i$, denoted $B_{i}(t)$, is a non-linear function of $q_{i}(t)$ and $v_{i}(t)$

$$
B_{i}(t)=V_{0}\left[k_{1}\left(1-q_{i}(t)\right)+k_{2}\left(1-\frac{q_{i}(t)}{v_{i}(t)}\right)+k_{3}\left(1-v_{i}(t)\right)\right]
$$

where $V_{0}$ represent the fraction of venous blood (deoxygenated) in resting-state, and $k_{1}, \quad{ }^{481}$ $k_{2}, k_{3}$ are kinetic constants. We used the same parameters as in Stephan et al. [42]: $\tau_{s}=0.65, \tau_{f}=0.41, \tau_{v}=0.98, \tau_{q}=0.98, \kappa=0.32, E_{0}=0.4, k_{1}=2.77, k_{2}=0.2$, $k_{3}=0.5$.

The system of differential equations 2 was solved with the Euler method, using an integration step of $1 \mathrm{~ms}$. The signals were band-pass filtered between 0.01 and $0.1 \mathrm{~Hz}$ with a 3rd order Bessel filter. These BOLD-like signals were used to build the functional connectivity (FC) matrices from which the subsequent analysis of functional network properties is performed using tools from graph theory.

\section{Global Phase Synchronization}

As a measure of global synchronization we calculated the Kuramoto order parameter $R(t)$ 43] of the EEG-like signals $\nu(t)$ derived from the Jansen \& Rit model. The raw signals were filtered with a 3rd order Bessel band-pass filter using their frequency of maximum power (usually between 4 and $10 \mathrm{~Hz}$ ) $\pm 3 \mathrm{~Hz}$. Then, the instantaneous phase $\phi(t)$ was obtained with the Hilbert transform.

The global phase synchrony is computed as:

$$
\bar{R}=\left\langle\left|\left\langle e^{j \phi_{i}(t)}\right\rangle_{N}\right|\right\rangle_{t}
$$

where $\phi_{i}(t)$ is the phase of the oscillator $i$ over time, $j=\sqrt{-1}$ the imaginary unit, $\mid$ denotes the module, \langle\rangle$_{N}$ denotes the average over all nodes, and \langle\rangle$_{t}$ the average over time. A value of $\bar{R}$ equal to 1 indicates perfect in-phase synchronization of all the set $N$ of oscillators, while a value equal to 0 indicates total asynchrony.

Metastability $\chi_{R}$, a measure of dynamical richness, is the variance of $R(t)[50$

$$
\chi_{R}=\operatorname{Var}\left(\left|\left\langle e^{j \phi_{i}(t)}\right\rangle_{N}\right|\right)
$$




\section{Signal-to-noise ratio}

The oscillation frequency $\omega_{i}$ of each node $i$ was computed as the peak frequency of its power spectral density function denoted $\operatorname{PSD}(\omega)$. This function was calculated using the Welch's method [75], with $20 \mathrm{~s}$ time windows overlapped by $50 \%$.

We calculated the average signal-to-noise ratio (SNR) overall raw signals, using the $P S D(\omega)$ function of each node and excluding the 2nd to 5 th harmonics $[76$. For a node $i$, the signal power, $P_{\text {signal }}$, was measured as the area under the curve of $P S D(\omega)$ within $\omega_{i} \pm 1 H z$. Noise power, $P_{\text {noise }}$, corresponds to the area under the curve of $P S D(\omega)$ outside the $\pm 1 H z$ window. Then the SNR was calculated as

$$
S N R=20 \log _{10} \frac{P_{\text {signal }}}{P_{\text {noise }}}
$$

The SNR was computed for each node $i$ and we reported the average overall nodes.

\section{Regularity}

We computed the regularity index in $20 \mathrm{~s}$ epochs of the raw EEG-like signals $\nu(t)$ as described by Malagarriga et al. [48]:

$$
\operatorname{Reg}=\left\langle h_{i}^{2 n d}(\tau)\right\rangle_{N},
$$

with $h_{i}^{2 n d}(\tau)$ being the second peak of the autocorrelation function for the node $i$ and $\langle\bullet\rangle_{N}$ the average over nodes. If the time series is purely periodic and has no noise, $h^{2 n d}(\tau)$ is equal to 1 (the signal is periodic and regular). Chaotic and noisy time series shift $h^{2 n d}(\tau)$ to lower values.

\section{Functional Connectivity and Graph Thresholding}

The static Functional Connectivity (sFC) matrices were built from pairwise Pearson's correlations of the entire BOLD-like time series. Instead of employing an absolute or proportional thresholding, we thresholded the sFCs matrices using Fourier transform (FT) surrogate data 77 to avoid the problem of introducing spurious correlations 78 . The FT algorithm uses a phase randomization process to destroy pairwise correlations, preserving the spectral properties of the signals (the surrogates have the same power spectrum as the original data). We generated 500 surrogates time series of the original set of BOLD-like signals, and then built the surrogates sFCs matrices. For each one of the $\left(n^{2}-n\right) / 2$ possible connectivity pairs (with $n=90$ ) we fitted a normal distribution of the surrogate values. Using these distributions we tested the hypothesis that a pairwise correlation is higher than chance (that is, the value is at the right of the surrogate distribution). To reject the null hypothesis, we selected a $p$-value equal to 0.05, and corrected for multiple comparisons with the FDR Benjamini-Hochberg procedure $[79$ to decrease the probability of make type I errors (false positives). The entries of the sFC matrix associated with a $p$-value less than 0.05 were set to 0 . The result is a thresholded, undirected, and weighted (with only positive values) $\mathrm{sFC}$ matrix.

\section{Integration and Segregation}

Integration and segregation were evaluated over the thresholded sFC matrices. We employed the weighted versions of transitivity 80 and global efficiency 81 to measure integration and segregation, respectively. A detailed description of the metrics used can be found in Rubinov \& Sporns [4]. The transitivity (similar to the average clustering coefficient) counts the fraction of triangular motifs surrounding the nodes (the 
equivalent of counting how many neighbors are also neighbors of each other), with the difference that it is normalized collectively. It is defined as

$$
T^{w}=\frac{\sum_{i \in N} 2 t_{i}^{w}}{\sum_{i \in N} k_{i}^{w}\left(k_{i}^{w}-1\right)}
$$

being $N$ the set of all nodes of the network with $n$ number of nodes, $t_{i}^{w}$ the geometric average of the triangles around the node $i$, and $k_{i}^{w}$ the node weighted degree. The supra-index $w$ is used to refer to the weighted versions of the topological network measures. On the other hand, the global efficiency is a measure of integration based on paths over the graph: it is defined as the inverse of the average shortest path length. This metric is computed as

$$
E^{w}=\frac{1}{n} \sum_{i \in N} \frac{\sum_{j \in N, j \neq i}\left(d_{i j}^{w}\right)^{-1}}{n-1}
$$

where $d_{i j}^{w}$ is the shortest path between the nodes $i$ and $j$.

We also calculated other two measures of integration and segregation: the participation coefficient $P C^{w}$ and modularity $Q^{w}$, respectively, both based on the detection of the network's communities 44]. The detection of so-called communities or network modules in the thresholded sFC matrix, was based on the Louvain's algorithm 82,83. The algorithm assigns a module to each node in a way that maximizes the modularity (3). We used the weighted version of the modularity [84] defined as

$$
Q^{w}=\frac{1}{l^{w}} \sum_{i, j \in N}\left[w_{i j}-\frac{k_{i}^{w} k_{j}^{w}}{l^{w}}\right] \delta_{m_{i}, m_{j}}
$$

where $w_{i j}$ is the weight of the link between $i$ and $j, l^{w}$ is the total number of weighted links of the network, $m_{i}\left(m_{j}\right)$ the module of the node $i(j)$. The Kronecker delta $\delta_{m_{i}, m_{j}}$ is equal to 1 when $m_{i}=m_{j}$ (that is, when two nodes belongs to the same module), and 0 otherwise. Because the Louvain's algorithm is stochastic, we employed the consensus clustering algorithm 85. We ran the Louvain's algorithm 200 times with the resolution parameter set to 1.0 (this parameter controls the size of the detected modules; larger values of this parameter allows the detection of smaller modules). Then, we built an agreement matrix $G$, in which an entry $G_{i j}$ indicates the proportion of partitions in which the pairs of nodes $(i, j)$ share the same module (so, the entries of $G$ are bounded between 0 and 1). Then, we applied an absolute threshold of 0.5 to the matrix $G$, and ran again the Louvain's algorithm 200 times using $G$ as input, producing a new consensus matrix $G^{\prime}$. This last step was repeated until convergence to an unique partition.

Finally, we computed the weighted version of the participation coefficient 86]. This metric quantifies, for each individual node, the strength of between-module connections respect to the within-module connections, and is defined as

$$
\left\langle P C^{w}\right\rangle_{N}=\frac{1}{n} \sum_{i \in N} P C_{i}^{w}=\frac{1}{n} \sum_{i \in N}\left(1-\sum_{m \in \mathcal{M}}\left(\frac{k_{i}^{w}(m)}{k_{i}^{w}}\right)^{2}\right)
$$

where $P C_{i}^{w}$ is the weighted participation coefficient for the node $i$, and $\left\langle P C^{w}\right\rangle_{N}$ is the average overall nodes. The functional network analysis was done in Python using the Brain Connectivity Toolbox 44 .

\section{Functional Connectivity Dynamics}

To test the hypothesis that dynamical variability peaks on the critical boundary, we performed a Functional Connectivity Dynamics (FCD) analysis over the filtered 
BOLD-like signals. The FCD matrix captures the evolution of FCs patterns and, consequently, the dynamical richness of the network 8,9$]$. We used the sliding window approach 8,51] depicted in the Fig 6. Window length was set to $100 \mathrm{~s}$ with a displacement of $2 \mathrm{~s}$ between consecutive windows (Fig 6A). The length was chosen on the basis of the lower limit of the band-pass filter $(0.01 \mathrm{~Hz})$, in order to minimize spurious correlations 87. For each window, a FC matrix was calculated from the pairwise Pearson's correlations of BOLD-like signals (neglecting negative values), thus we obtained 251 weighted and undirected FCs matrices from the $600 \mathrm{~s}$ simulated BOLD-like signals (Fig 6B).

The upper triangle of each FC matrix is unfolded to make a vector, and the FCD is built by calculating the Clarkson angular distance $\lambda(x, y)=\frac{1}{\sqrt{2}}\left\|\frac{x}{\|x\|}-\frac{y}{\|y\|}\right\| \mid 88$ between each pair of FCs Fig 6C)

$$
F C D_{i j}=\lambda\left(F C\left(t_{i}\right), F C\left(t_{j}\right)\right)
$$

The variance of the values in the upper triangle of the FCD, with an offset of $\tau=100 \mathrm{~s}$ from the diagonal (e.g., the variance of the histograms of Fig 6D), is taken as a measure of dynamical richness [51].

The speed of the FCD was measured as described by Battaglia et al. [52. We computed the histogram of $\mathrm{FCD}$ values through a straight line from $\operatorname{FCD}(\tau, 0)$ to $\mathrm{FCD}\left(t_{\max }, t_{\max }-\tau\right)$, with $t_{\max }=600 \mathrm{~s}$ as the total time-length of the signals and $\tau=100 \mathrm{~s}$. The median of the histogram distribution corresponded to the typical FCD speed $d_{t y p}$. Values closer to 1 indicate a constant switching of states, and values closer to 0 correspond to stable FCs patterns.

\section{Supporting information}

S1 Fig. Alternative measures of network segregation and integration in the $(\boldsymbol{\alpha}, \boldsymbol{\beta})$ parameter space. A) Mean participation coefficient $P C^{w}$ (integration) and transitivity $T^{w}$ (segregation). B-C) Transitions in the direction of $\alpha$ and $\beta$ axes. Dashed lines represent critical points.

S2 Fig. Signal and network features in the $\left(\alpha, C_{1}\right)$ parameter space. A) Average phase synchrony $\bar{R}$, mean oscillatory frequency $\omega$ of EEG-like signals, global efficiency $E^{w}$ (integration) and modularity $Q^{w}$ (segregation) of the graphs derived from the sFCs of the BOLD-like signals. B) Transitions in the direction of $\alpha$ axis, for a fixed $C_{1}=0$. C) Transitions in the direction of $C_{1}$ axis, for a fixed $\alpha=0.5$. Dashed lines represent critical points.

S3 Fig. Alternative measures of network segregation and integration in the $\left(\alpha, r_{0}\right)$ parameter space. A-B) Mean participation coefficient $P C^{w}$ (integration) and transitivity $T^{w}$ (segregation) with $\left.\mathbf{A}\right) \beta=0$ and $\left.\mathbf{B}\right) \beta=0.4$. C-D) Transitions in the direction of $\alpha$ and $r_{0}$ axes. Dashed lines represent critical points.

S4 Fig. Simultaneously effect of $\alpha, \beta$ and $r_{0}$ in signal and network features. A) Average phase synchrony $\bar{R}$, mean oscillatory frequency $\omega$ of EEG-like signals, global efficiency $E^{w}$ (integration) and modularity $Q^{w}$ (segregation) of the graphs derived from the sFCs of the BOLD-like signals. B) Transitions in the direction of $\alpha$ axis, for a fixed $r_{0}=0.5 \mathrm{mV}^{-1}$. C) Transitions in the direction of $r_{0}$ axis, for a fixed $\alpha=0.5$. Dashed lines represent critical points. Response gains change in parallel following the relationship $\beta=0.35 \alpha$. 


\section{Acknowledgments}

We thank to Gustavo Deco, from Pompeu Fabra University (UPF, Barcelona, Spain), who kindly provided the anatomical connectivity matrix used in the model. We also want to thank to Chiayu Chiu and Andrés Chávez, from Centro Interdisciplinario de Neurociencia de Valparaíso (CINV, Valparaíso, Chile), for their feedback and suggestions about the manuscript. This work was supported by Fondecyt Grants 1181076 (to PO) and 11181072 (to RC) and the Advanced Center for Electrical and Electronic Engineering (FB0008 ANID, Chile). The Centro Interdisciplinario de Neurociencia de Valparaíso (CINV) is a Millenium Institute supported by the Millennium Scientific Initiative (ANID). CC is funded by Beca Doctorado Nacional ANID 2018- 21180995.

\section{References}

1. Tononi G, Sporns O, Edelman GM. A measure for brain complexity: relating functional segregation and integration in the nervous system. Proceedings of the National Academy of Sciences. 1994;91(11):5033-5037. doi:https://doi.org/10.1073/pnas.91.11.5033.

2. Cohen JR, D'Esposito M. The segregation and integration of distinct brain networks and their relationship to cognition. Journal of Neuroscience. 2016;36(48):12083-12094. doi:https://doi.org/10.1523/JNEUROSCI.2965-15.2016.

3. Shine JM, Bissett PG, Bell PT, Koyejo O, Balsters JH, Gorgolewski KJ, et al. The dynamics of functional brain networks: integrated network states during cognitive task performance. Neuron. 2016;92(2):544-554. doi:https://doi.org/10.1016/j.neuron.2016.09.018.

4. Shine JM, Breakspear M, Bell PT, Martens KAE, Shine R, Koyejo O, et al. Human cognition involves the dynamic integration of neural activity and neuromodulatory systems. Nature neuroscience. 2019;22(2):289-296. doi:https://doi.org/10.1038/s41593-018-0312-0.

5. Kelso JS. Multistability and metastability: understanding dynamic coordination in the brain. Philosophical Transactions of the Royal Society B: Biological Sciences. 2012;367(1591):906-918. doi:https://doi.org/10.1098/rstb.2011.0351.

6. Tognoli E, Kelso JS. The metastable brain. Neuron. 2014;81(1):35-48. doi:https://doi.org/10.1016/j.neuron.2013.12.022.

7. Sporns O. Network attributes for segregation and integration in the human brain. Current opinion in neurobiology. 2013;23(2):162-171. doi:https://doi.org/10.1016/j.conb.2012.11.015.

8. Hansen EC, Battaglia D, Spiegler A, Deco G, Jirsa VK. Functional connectivity dynamics: modeling the switching behavior of the resting state. Neuroimage. 2015;105:525-535. doi:https://doi.org/10.1016/j.neuroimage.2014.11.001.

9. Cabral J, Kringelbach ML, Deco G. Functional connectivity dynamically evolves on multiple time-scales over a static structural connectome: Models and mechanisms. NeuroImage. 2017;160:84-96. doi:https://doi.org/10.1016/j.neuroimage.2017.03.045. 
10. Shine JM. Neuromodulatory influences on integration and segregation in the brain. Trends in cognitive sciences.

2019;doi:https://doi.org/10.1016/j.tics.2019.04.002.

11. Thiele A, Bellgrove MA. Neuromodulation of attention. Neuron. 2018;97(4):769-785. doi:https://doi.org/10.1016/j.neuron.2018.01.008.

12. Herrero JL, Roberts M, Delicato LS, Gieselmann MA, Dayan P, Thiele A. Acetylcholine contributes through muscarinic receptors to attentional modulation in V1. Nature. 2008;454(7208):1110-1114. doi:https://doi.org/10.1038/nature07141.

13. Thiele A, Herrero JL, Distler C, Hoffmann KP. Contribution of cholinergic and GABAergic mechanisms to direction tuning, discriminability, response reliability, and neuronal rate correlations in macaque middle temporal area. Journal of Neuroscience. 2012;32(47):16602-16615. doi:https://doi.org/10.1523/JNEUROSCI.0554-12.2012.

14. Foote S, Aston-Jones G, Bloom F. Impulse activity of locus coeruleus neurons in awake rats and monkeys is a function of sensory stimulation and arousal. Proceedings of the National Academy of Sciences. 1980;77(5):3033-3037. doi:https://doi.org/10.1073/pnas.77.5.3033.

15. Waterhouse BD, Sessler FM, Jung-Tung C, Woodward DJ, Azizi SA, Moises HC. New evidence for a gating action of norepinephrine in central neuronal circuits of mammalian brain. Brain research bulletin. 1988;21(3):425-432. doi:https://doi.org/10.1016/0361-9230(88)90154-2.

16. Waterhouse BD, Moises HC, Woodward DJ. Phasic activation of the locus coeruleus enhances responses of primary sensory cortical neurons to peripheral receptive field stimulation. Brain research. 1998;790(1-2):33-44. doi:https://doi.org/10.1016/s0006-8993(98)00117-6.

17. Servan-Schreiber D, Printz H, Cohen JD. A network model of catecholamine effects: gain, signal-to-noise ratio, and behavior. Science. 1990;249(4971):892-895. doi:https://doi.org/10.1126/science.2392679.

18. Shine JM, Aburn MJ, Breakspear M, Poldrack RA. The modulation of neural gain facilitates a transition between functional segregation and integration in the brain. Elife. 2018;7:e31130. doi:https://doi.org/10.7554/eLife.31130.001.

19. Bentley P, Driver J, Dolan RJ. Cholinergic modulation of cognition: insights from human pharmacological functional neuroimaging. Progress in neurobiology. 2011;94(4):360-388. doi:https://doi.org/10.1016/j.pneurobio.2011.06.002.

20. Xiang Z, Huguenard JR, Prince DA. Cholinergic switching within neocortical inhibitory networks. Science. 1998;281(5379):985-988. doi:https://doi.org/10.1126/science.281.5379.985.

21. Kimura F. Cholinergic modulation of cortical function: a hypothetical role in shifting the dynamics in cortical network. Neuroscience research. 2000;38(1):19-26. doi:https://doi.org/10.1016/s0168-0102(00)00151-6.

22. Picciotto MR, Higley MJ, Mineur YS. Acetylcholine as a neuromodulator: cholinergic signaling shapes nervous system function and behavior. Neuron. 2012;76(1):116-129. doi:https://doi.org/10.1016/j.neuron.2012.08.036. 
23. Poorthuis RB, Bloem B, Schak B, Wester J, de Kock CP, Mansvelder HD. Layer-specific modulation of the prefrontal cortex by nicotinic acetylcholine receptors. Cerebral cortex. 2013;23(1):148-161. doi:https://doi.org/10.1093/cercor/bhr390.

24. Hasselmo M, Giocomo L. Cholinergic modulation of cortical function. Journal of Molecular Neuroscience. 2006;30(1-2):133. doi:https://doi.org/10.1385/JMN:30:1:133.

25. Kruglikov I, Rudy B. Perisomatic GABA release and thalamocortical integration onto neocortical excitatory cells are regulated by neuromodulators. Neuron. 2008;58(6):911-924. doi:https://doi.org/10.1016/j.neuron.2008.04.024.

26. Fanselow EE, Richardson KA, Connors BW. Selective, state-dependent activation of somatostatin-expressing inhibitory interneurons in mouse neocortex. Journal of neurophysiology. 2008;100(5):2640-2652. doi:https://doi.org/10.1152/jn.90691.2008.

27. Arroyo S, Bennett C, Aziz D, Brown SP, Hestrin S. Prolonged disynaptic inhibition in the cortex mediated by slow, non- $\alpha 7$ nicotinic excitation of a specific subset of cortical interneurons. Journal of Neuroscience. 2012;32(11):3859-3864. doi:https://doi.org/10.1523/JNEUROSCI.0115-12.2012.

28. Deco G, Kringelbach ML. Great expectations: using whole-brain computational connectomics for understanding neuropsychiatric disorders. Neuron. 2014;84(5):892-905. doi:https://doi.org/10.1016/j.neuron.2014.08.034.

29. Lynn CW, Bassett DS. The physics of brain network structure, function and control. Nature Reviews Physics. 2019;1(5):318. doi:https://doi.org/10.1038/s42254-019-0040-8.

30. Deco G, Tononi G, Boly M, Kringelbach ML. Rethinking segregation and integration: contributions of whole-brain modelling. Nature Reviews Neuroscience. 2015;16(7):430-439. doi:https://doi.org/10.1038/nrn3963.

31. Lord LD, Stevner AB, Deco G, Kringelbach ML. Understanding principles of integration and segregation using whole-brain computational connectomics: implications for neuropsychiatric disorders. Philosophical Transactions of the Royal Society A: Mathematical, Physical and Engineering Sciences. 2017;375(2096):20160283. doi:https://doi.org/10.1098/rsta.2016.0283.

32. Jansen BH, Rit VG. Electroencephalogram and visual evoked potential generation in a mathematical model of coupled cortical columns. Biological cybernetics. 1995;73(4):357-366. doi:https://doi.org/10.1007/bf00199471.

33. Chakravarthy N, Sabesan S, Tsakalis K, Iasemidis L. Controlling epileptic seizures in a neural mass model. Journal of Combinatorial Optimization. 2009;17(1):98-116. doi:https://doi.org/10.1007/s10878-008-9182-9.

34. Spiegler A, Knösche TR, Schwab K, Haueisen J, Atay FM. Modeling brain resonance phenomena using a neural mass model. PLoS computational biology. 2011;7(12). doi:https://doi.org/10.1371/journal.pcbi.1002298.

35. Stefanovski L, Triebkorn JP, Spiegler A, Diaz-Cortes MA, Solodkin A, Jirsa V, et al. Linking molecular pathways and large-scale computational modeling to assess candidate disease mechanisms and pharmacodynamics in Alzheimer's disease. Frontiers in computational neuroscience. 2019;13:54. doi:https://doi.org/10.3389/fncom.2019.00054. 
36. Silberberg G, Markram H. Disynaptic inhibition between neocortical pyramidal cells mediated by Martinotti cells. Neuron. 2007;53(5):735-746.

doi:https://doi.org/10.1016/j.neuron.2007.02.012.

37. Fino E, Packer AM, Yuste R. The logic of inhibitory connectivity in the neocortex. The Neuroscientist. 2013;19(3):228-237.

doi:https://doi.org/10.1177/1073858412456743.

38. Deco G, Cruzat J, Cabral J, Knudsen GM, Carhart-Harris RL, Whybrow PC, et al. Whole-brain multimodal neuroimaging model using serotonin receptor maps explains non-linear functional effects of LSD. Current biology. 2018;28(19):3065-3074. doi:https://doi.org/10.1016/j.cub.2018.07.083.

39. Tzourio-Mazoyer N, Landeau B, Papathanassiou D, Crivello F, Etard O, Delcroix $\mathrm{N}$, et al. Automated anatomical labeling of activations in SPM using a macroscopic anatomical parcellation of the MNI MRI single-subject brain. Neuroimage. 2002;15(1):273-289. doi:https://doi.org/10.1006/nimg.2001.0978.

40. Gilbert CD, Hirsch JA, Wiesel TN. Lateral interactions in visual cortex. In: Cold Spring Harbor symposia on quantitative biology. vol. 55. Cold Spring Harbor Laboratory Press; 1990. p. 663-677.

41. McGuire BA, Gilbert CD, Rivlin PK, Wiesel TN. Targets of horizontal connections in macaque primary visual cortex. Journal of Comparative Neurology. 1991;305(3):370-392. doi:https://doi.org/10.1002/cne.903050303.

42. Stephan KE, Weiskopf N, Drysdale PM, Robinson PA, Friston KJ. Comparing hemodynamic models with DCM. Neuroimage. 2007;38(3):387-401. doi:https://doi.org/10.1016/j.neuroimage.2007.07.040.

43. Acebrón JA, Bonilla LL, Vicente CJP, Ritort F, Spigler R. The Kuramoto model: A simple paradigm for synchronization phenomena. Reviews of modern physics. 2005;77(1):137. doi:https://doi.org/10.1103/RevModPhys.77.137.

44. Rubinov M, Sporns O. Complex network measures of brain connectivity: uses and interpretations. Neuroimage. 2010;52(3):1059-1069. doi:https://doi.org/10.1016/j.neuroimage.2009.10.003.

45. Chialvo DR. Emergent complex neural dynamics. Nature physics. 2010;6(10):744-750. doi:https://doi.org/10.1038/nphys1803.

46. Nordenfelt A, Wagemakers A, Sanjuán MA. Frequency dispersion in the time-delayed Kuramoto model. Physical Review E. 2014;89(3):032905. doi:https://doi.org/10.1103/PhysRevE.89.032905.

47. Lea-Carnall CA, Montemurro MA, Trujillo-Barreto NJ, Parkes LM, El-Deredy W. Cortical resonance frequencies emerge from network size and connectivity. PLoS computational biology. 2016;12(2). doi:https://doi.org/10.1371/journal.pcbi.1004740.

48. Malagarriga D, Villa AE, Garcia-Ojalvo J, Pons AJ. Mesoscopic segregation of excitation and inhibition in a brain network model. PLoS computational biology. 2015;11(2). doi:https://doi.org/10.1371/journal.pcbi.1004007.

49. Buzsáki G, Draguhn A. Neuronal oscillations in cortical networks. science. 2004;304(5679):1926-1929. doi:https://doi.org/10.1126/science.1099745. 
50. Wildie M, Shanahan M. Metastability and chimera states in modular delay and pulse-coupled oscillator networks. Chaos: An Interdisciplinary Journal of Nonlinear Science. 2012;22(4):043131. doi:https://doi.org/10.1063/1.4766592.

51. Orio P, Gatica M, Herzog R, Maidana JP, Castro S, Xu K. Chaos versus noise as drivers of multistability in neural networks. Chaos: An Interdisciplinary Journal of Nonlinear Science. 2018;28(10):106321. doi:https://doi.org/10.1063/1.5043447.

52. Battaglia D, Boudou T, Hansen EC, Lombardo D, Chettouf S, Daffertshofer A, et al. Dynamic Functional Connectivity between Order and Randomness and its Evolution across the Human Adult Lifespan. bioRxiv. 2020; p. 107243. doi:https://doi.org/10.1016/j.neuroimage.2020.117156.

53. Lee WH, Frangou S. Linking functional connectivity and dynamic properties of resting-state networks. Scientific reports. 2017;7(1):1-10. doi:https://doi.org/10.1038/s41598-017-16789-1.

54. Shafiei G, Zeighami Y, Clark CA, Coull JT, Nagano-Saito A, Leyton M, et al. Dopamine signaling modulates the stability and integration of intrinsic brain networks. Cerebral Cortex. 2019;29(1):397-409. doi:https://doi.org/10.1093/cercor/bhy264.

55. Scharinger C, Soutschek A, Schubert T, Gerjets P. Comparison of the working memory load in n-back and working memory span tasks by means of EEG frequency band power and P300 amplitude. Frontiers in human neuroscience. 2017;11:6. doi:https://doi.org/10.3389/fnhum.2017.00006.

56. Harmony T. The functional significance of delta oscillations in cognitive processing. Frontiers in integrative neuroscience. 2013;7:83. doi:https://doi.org/10.3389/fnint.2013.00083.

57. Lord LD, Expert P, Fernandes HM, Petri G, Van Hartevelt TJ, Vaccarino F, et al. Insights into brain architectures from the homological scaffolds of functional connectivity networks. Frontiers in systems neuroscience. 2016;10:85. doi:https://doi.org/10.3389/fnsys.2016.00085.

58. Deco G, Ponce-Alvarez A, Hagmann P, Romani GL, Mantini D, Corbetta M. How local excitation-inhibition ratio impacts the whole brain dynamics. Journal of Neuroscience. 2014;34(23):7886-7898. doi:https://doi.org/10.1523/JNEUROSCI.5068-13.2014.

59. Wylie KP, Rojas DC, Tanabe J, Martin LF, Tregellas JR. Nicotine increases brain functional network efficiency. Neuroimage. 2012;63(1):73-80. doi:https://doi.org/10.1016/j.neuroimage.2012.06.079.

60. Valentine G, Sofuoglu M. Cognitive effects of nicotine: recent progress. Current neuropharmacology. 2018;16(4):403-414. doi:https://doi.org/10.2174/1570159X15666171103152136.

61. Spiegler A, Kiebel SJ, Atay FM, Knösche TR. Bifurcation analysis of neural mass models: Impact of extrinsic inputs and dendritic time constants. NeuroImage. 2010;52(3):1041-1058. doi:https://doi.org/10.1016/j.neuroimage.2009.12.081.

62. Shew WL, Yang H, Yu S, Roy R, Plenz D. Information capacity and transmission are maximized in balanced cortical networks with neuronal avalanches. Journal of neuroscience. 2011;31(1):55-63. doi:https://doi.org/10.1523/JNEUROSCI.4637-10.2011. 
63. Shen EH, Overly CC, Jones AR. The Allen Human Brain Atlas: comprehensive gene expression mapping of the human brain. Trends in neurosciences. 2012;35(12):711-714. doi:https://doi.org/10.1016/j.tins.2012.09.005.

64. Marder E. Neuromodulation of neuronal circuits: back to the future. Neuron. 2012;76(1):1-11. doi:https://doi.org/10.1016/j.neuron.2012.09.010.

65. Kringelbach ML, Cruzat J, Cabral J, Knudsen GM, Carhart-Harris R, Whybrow $\mathrm{PC}$, et al. Dynamic coupling of whole-brain neuronal and neurotransmitter systems. Proceedings of the National Academy of Sciences. 2020;117(17):9566-9576. doi:https://doi.org/10.1073/pnas.1921475117.

66. Wendling F, Bartolomei F, Bellanger J, Chauvel P. Epileptic fast activity can be explained by a model of impaired GABAergic dendritic inhibition. European Journal of Neuroscience. 2002;15(9):1499-1508. doi:https://doi.org/10.1046/j.1460-9568.2002.01985.x.

67. Rosas FE, Mediano PA, Gastpar M, Jensen HJ. Quantifying high-order interdependencies via multivariate extensions of the mutual information. Physical Review E. 2019;100(3):032305. doi:https://doi.org/10.1103/PhysRevE.100.032305.

68. Gatica M, Cofre R, Mediano PA, Rosas FE, Orio P, Diez I, et al. High-order interdependencies in the aging brain. bioRxiv. 2020;doi:https://doi.org/10.1101/2020.03.17.995886.

69. Petri G, Expert P, Turkheimer F, Carhart-Harris R, Nutt D, Hellyer PJ, et al. Homological scaffolds of brain functional networks. Journal of The Royal Society Interface. 2014;11(101):20140873. doi:https://doi.org/10.1098/rsif.2014.0873.

70. Geniesse C, Sporns O, Petri G, Saggar M. Generating dynamical neuroimaging spatiotemporal representations (DyNeuSR) using topological data analysis. Network Neuroscience. 2019;3(3):763-778. doi:https://doi.org/10.1162/netn ${ }_{a 0} 0093$.

71. Griffa A, Baumann PS, Thiran JP, Hagmann P. Structural connectomics in brain diseases. Neuroimage. 2013;80:515-526. doi:https://doi.org/10.1016/j.neuroimage.2013.04.056.

72. Brier MR, Thomas JB, Fagan AM, Hassenstab J, Holtzman DM, Benzinger TL, et al. Functional connectivity and graph theory in preclinical Alzheimer's disease. Neurobiology of aging. 2014;35(4):757-768. doi:https://doi.org/10.1016/j.neurobiolaging.2013.10.081.

73. Hadley JA, Kraguljac NV, White DM, Ver Hoef L, Tabora J, Lahti AC. Change in brain network topology as a function of treatment response in schizophrenia: a longitudinal resting-state fMRI study using graph theory. npj Schizophrenia. 2016;2(1):1-7. doi:https://doi.org/10.1038/npjschz.2016.14.

74. Birn RM, Molloy EK, Patriat R, Parker T, Meier TB, Kirk GR, et al. The effect of scan length on the reliability of resting-state fMRI connectivity estimates. Neuroimage. 2013;83:550-558. doi:https://doi.org/10.1016/j.neuroimage.2013.05.099.

75. Welch P. The use of fast Fourier transform for the estimation of power spectra: a method based on time averaging over short, modified periodograms. IEEE Transactions on audio and electroacoustics. 1967;15(2):70-73. doi:https://doi.org/10.1109/TAU.1967.1161901. 
76. Schultz SR. Signal-to-noise ratio in neuroscience. Scholarpedia. 2007;2(6):2046. doi:https://doi.org/doi:10.4249/scholarpedia.2046.

77. Lancaster G, Iatsenko D, Pidde A, Ticcinelli V, Stefanovska A. Surrogate data for hypothesis testing of physical systems. Physics Reports. 2018;748:1-60. doi:https://doi.org/10.1016/j.physrep.2018.06.001.

78. Fornito A, Zalesky A, Breakspear M. Graph analysis of the human connectome: promise, progress, and pitfalls. Neuroimage. 2013;80:426-444. doi:https://doi.org/10.1016/j.neuroimage.2013.04.087.

79. Benjamini Y, Hochberg Y. Controlling the false discovery rate: a practical and powerful approach to multiple testing. Journal of the Royal statistical society: series B (Methodological). 1995;57(1):289-300. doi:https://doi.org/10.1111/j.2517-6161.1995.tb02031.x.

80. Newman ME. The structure and function of complex networks. SIAM review. 2003;45(2):167-256. doi:https://doi.org/10.1137/S003614450342480.

81. Latora V, Marchiori M. Efficient behavior of small-world networks. Physical review letters. 2001;87(19):198701. doi:https://doi.org/10.1103/PhysRevLett.87.198701.

82. Newman ME. Modularity and community structure in networks. Proceedings of the national academy of sciences. 2006;103(23):8577-8582. doi:https://doi.org/10.1073/pnas.0601602103.

83. Blondel VD, Guillaume JL, Lambiotte R, Lefebvre E. Fast unfolding of communities in large networks. Journal of statistical mechanics: theory and experiment. 2008;2008(10):P10008. doi:https://doi.org/10.1088/1742-5468/2008/10/P10008.

84. Newman ME. Analysis of weighted networks. Physical review E. 2004;70(5):056131. doi:https://doi.org/10.1103/PhysRevE.70.056131.

85. Lancichinetti A, Fortunato S. Consensus clustering in complex networks. Scientific reports. 2012;2:336. doi:https://doi.org/10.1038/srep00336.

86. Guimera R, Amaral LAN. Cartography of complex networks: modules and universal roles. Journal of Statistical Mechanics: Theory and Experiment. 2005;2005(02):P02001. doi:https://doi.org/10.1088/1742-5468/2005/02/P02001.

87. Leonardi N, Van De Ville D. On spurious and real fluctuations of dynamic functional connectivity during rest. Neuroimage. 2015;104:430-436. doi:https://doi.org/10.1016/j.neuroimage.2014.09.007.

88. Clarkson JA. Uniformly convex spaces. Transactions of the American Mathematical Society. 1936;40(3):396-414. doi:https://doi.org/10.2307/1989630. 